Linköping Studies in Science and Technology Thesis No. 1678

\title{
Online Learning for Robot Vision
}

\author{
Kristoffer Öfjäll
}

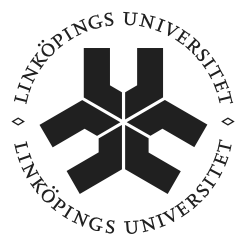

\section{Linköping University}

INSTITUTE OF TECHNOLOGY

Department of Electrical Engineering

Linköpings universitet, SE-581 83 Linköping, Sweden

Linköping August 2014 
Online Learning for Robot Vision

\author{
(c) 2014 Kristoffer Öfjäll \\ Department of Electrical Engineering \\ Linköping University \\ SE-581 83 Linköping \\ Sweden
}

ISBN 978-91-7519-228-4

ISSN 0280-7971 


\section{Abstract}

In tele-operated robotics applications, the primary information channel from the robot to its human operator is a video stream. For autonomous robotic systems however, a much larger selection of sensors is employed, although the most relevant information for the operation of the robot is still available in a single video stream. The issue lies in autonomously interpreting the visual data and extracting the relevant information, something humans and animals perform strikingly well. On the other hand, humans have great difficulty expressing what they are actually looking for on a low level, suitable for direct implementation on a machine. For instance objects tend to be already detected when the visual information reaches the conscious mind, with almost no clues remaining regarding how the object was identified in the first place. This became apparent already when Seymour Papert gathered a group of summer workers to solve the computer vision problem 48 years ago [35].

Artificial learning systems can overcome this gap between the level of human visual reasoning and low-level machine vision processing. If a human teacher can provide examples of what to be extracted and if the learning system is able to extract the gist of these examples, the gap is bridged. There are however some special demands on a learning system for it to perform successfully in a visual context. First, low level visual input is often of high dimensionality such that the learning system needs to handle large inputs. Second, visual information is often ambiguous such that the learning system needs to be able to handle multi modal outputs, i.e. multiple hypotheses. Typically, the relations to be learned are non-linear and there is an advantage if data can be processed at video rate, even after presenting many examples to the learning system. In general, there seems to be a lack of such methods.

This thesis presents systems for learning perception-action mappings for robotic systems with visual input. A range of problems are discussed, such as vision based autonomous driving, inverse kinematics of a robotic manipulator and controlling a dynamical system. Operational systems demonstrating solutions to these problems are presented. Two different approaches for providing training data are explored, learning from demonstration (supervised learning) and explorative learning (self-supervised learning). A novel learning method fulfilling the stated demands is presented. The method, qHebb, is based on associative Hebbian learning on data in channel representation. Properties of the method are demonstrated on a vision-based autonomously driving vehicle, where the system learns to directly map low-level image features to control signals. After an initial training period, the system seamlessly continues autonomously. In a quantitative evaluation, the proposed online learning method performed comparably with state of the art batch learning methods. 


\section{Acknowledgments}

First I would like to acknowledge the effort of the portable air conditioning unit, keeping the office at a reasonable temperature during the most intense period of writing. In general I would like to thank everyone in the set of people who have influenced my life in any way, however, since its cardinality is far too great and since its boundaries are too fuzzy, I will need to settle with a few samples.

I will start by mentioning all friends who have supported me during good times and through bad times, friends who have accompanied me during my many years at different educational institutions, who have managed to make even the exam periods enjoyable. Interesting are all the strange places where, apparently, it is possible to study for exams. I would also like to thank all friends for all the fun during activities collectively described as not studying. Especially I would like to mention everyone who have joined me, and invited me to join, on all adventures, ranging from the highest summits of northern Europe down to caves and abandoned mines deep below the surface.

Concerning people who have had a more direct influence on the existence of the following text I would like to mention: Anders Klang, for (unintentionally, I believe) making me go for a master's degree. Johan Hedborg, for (more intentionally) convincing me to continue postgraduate. Everyone at CVL and all the people I have met during conferences and the like, for great discussions and inspiration. My main supervisor Michael Felsberg, for providing great guidance through the sometimes murky waters of science and for being a seemingly infinite source of ideas and motivation.

Finally, I would like to thank my family for unlimited support in any matter.

This work has been supported by EC's 7th Framework Programme, grant agreement 247947 (GARNICS), by SSF through a grant for the project CUAS, by VR through a grant for the project ETT, through the Strategic Areas for ICT research CADICS and ELLIIT.

Kristoffer Öfjäll August 2014 


\section{Contents}

I Background Theory 1

1 Introduction 3

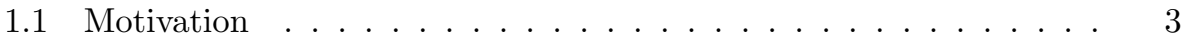

1.2 Outline Part I: Background Theory . . . . . . . . . . . . . . . . . . 4

1.3 Outline Part II: Included Publications . . . . . . . . . . . . . . 5

2 Perception-Action Mappings 9

2.1 Vision Based Autonomous Driving . . . . . . . . . . . . . . . 9

2.2 Inverse Kinematics . . . . . . . . . . . . . . . . . . . . . . . . . . . 11

2.3 Dynamic System Control . . . . . . . . . . . . . . . . 12

3 Learning Methods $\quad \mathbf{1 5}$

3.1 Classification of Learning Methods . . . . . . . . . . . . . . . . 16

3.1 .1 Online Learning . . . . . . . . . . . . . . . . . 16

3.1 .2 Active Learning . . . . . . . . . . . . . . . . . . . . . . . . 16

3.1.3 Multi Modal Learning . . . . . . . . . . . . . . . . 17

3.2 Training Data Source . . . . . . . . . . . . . . . . . . . . . 18

3.2.1 Explorative Learning . . . . . . . . . . . . . . . . . . 18

3.2.2 Learning from Demonstration . . . . . . . . . . . . . . . 19

3.2.3 Reinforcement Learning . . . . . . . . . . . . . . . . 20

3.3 Locally Weighted Projection Regression . . . . . . . . . . . . . . 20

3.3.1 High Dimensional Issues . . . . . . . . . . . . . . . . . . . . 21

3.4 Random Forest Regression . . . . . . . . . . . . . . . . . . . . 21

3.5 Hebbian Learning . . . . . . . . . . . . . . . . . . . . . . . . . . . . . . . . . . . . 22

3.6 Associative Learning . . . . . . . . . . . . . . . . . 24

4 Representation $\quad 27$

4.1 The Channel Representation . . . . . . . . . . . . . . . . . 28

4.1.1 Channel Encoding . . . . . . . . . . . . . . . . . 29

4.1.2 Views of the Channel Vector ............. . . 31

4.1.3 The $\cos ^{2}$ Basis Function . . . . . . . . . . . . . . . 32

4.1.4 Robust Decoding . . . . . . . . . . . . . . . . 32

4.2 Mixtures of Local Models . . . . . . . . . . . . . . . . . . . . . . . . . . . 34

4.2.1 Tree Based Sectioning . . . . . . . . . . . . . . . 34

4.2.2 Weighted Local Models . . . . . . . . . . . . 34 
5 Experiment Highlights $\quad \mathbf{3 7}$

5.1 Vision Based Autonomous Driving . . . . . . . . . . . . . . 37

5.1.1 Random Forest Regression . . . . . . . . . . . . . . 38

5.1 .2 Locally Weighted Projection Regression . . . . . . . . . 38

5.1.3 Extended Hebbian Associative Learning: qHebb . . . . . . 43

5.2 Inverse Kinematics . . . . . . . . . . . . . . . . . . . . . . 44

5.2.1 Seven DoF Robotic Manipulator . . . . . . . . . . . . . 44

5.2 .2 Synthetic Evaluation . . . . . . . . . . . . . . . 47

5.3 Dynamic System Control _. . . . . . . . . . . . . . . . . 48

5.3.1 Experiments in Spatially Invariant Maze . . . . . . . . . . 52

5.3 .2 Experiment in Real Maze . . . . . . . . . . . . . . 53

6 Conclusion and Future Research $\quad 57$

II Publications $\quad 63$

A Autonomous Navigation and Sign Detector Learning 65

B Online Learning of Vision-Based Robot Control during Autonomous $\begin{array}{ll}\text { Operation } & 75\end{array}$

C Biologically Inspired Online Learning of Visual Autonomous Driv$\begin{array}{ll}\text { ing } & 97\end{array}$

D Combining Vision, Machine Learning and Automatic Control to Play the Labyrinth Game 


\section{Part I}

\section{Background Theory}





\section{Chapter 1}

\section{Introduction}

This thesis is about robot vision. Robot control requires extracting information about the environment and there are two approaches to facilitate acquisition of information. One is to add more sensors, the other is to extract more information from the sensor data already available. A tendency to use many sensors can be seen in the autonomous cars of the DARPA challenges, where the cost of the sensors is several times higher than the cost of the car itself.

There is a wide range of different sensors in terms of the amount of information provided by each measurement, however, this seems to be inversely proportional to the complexity of interpreting said measurement. The simplest sensors measure one particular entity, the rotational speed of a wheel or the distance to the closest object along a particular line. Using only these types of sensors the system usually misses the big picture.

\subsection{Motivation}

For robotics applications, a single camera alone often provides enough information for successfully completing the task at hand. Numerous tele-operated robots and vehicles where the only feedback to the operator is a video stream are examples of this. For autonomous systems on the other hand, where the visual processing capabilities and experience of a human operator is not available, sensors with simpler but also more easily interpretable output data are more common.

The challenge lies in extracting the relevant information from visual data by an autonomous system. It may even be troublesome for a human to provide the system with a useful description of what relevant information is. In general, humans tend to reason at a higher perceptual level. An instruction such as "follow the road" is usually clear to a human operator. However, for an autonomous vehicle, a suitable instruction is something more similar to "adjust this voltage such that these patterns of areas with, in general, slightly higher light intensity in a particular pattern stays at these regions of the image". The latter is hard for a human to understand, and at the same time it only covers a small subset of the situations where the former instruction is applicable. 
In such situations, machine learning in general, and learning from demonstration in particular, can bridge the gap between the levels of reasoning of a human operator and an autonomous system. By letting a human operator control the vehicle, a collection of images with corresponding appropriate actions, i.e. training data, is gathered. From this training data, an algorithm may extract informative intensity patterns and the corresponding control signals. Through this, both the human operator and the autonomous system can operate at their own preferred level of perception.

Machine learning from visual input poses many challenges which have kept researchers busy for decades. Some particular challenges will be presented here, the first is the ambiguity of visual perceptions. These are known to humans as optical illusions. One example is the middle image of Fig. 1.1. The interpretation is multi-modal, there are multiple hypotheses regarding the interpretation of what is seen. From a learning systems point of view, the algorithm and representation should be general enough to allow multiple hypoteses, or in mathematical terms, there should be a possibility to map a certain input to several different outputs.

Further, the resolution of cameras steadily increases. Visual learning systems must be capable of handling high dimensional data, each image is a vector in an order of a million dimensional space. Processing of data should also be fast. Online learning systems, which can process training data at the rate at which it is produced, have clear advantages for the human operator. One common question is how much training data is required. An online learning system can provide direct feedback to the operator regarding the training progress and when enough training data has been collected. For offline, or batch learning, systems, the operator will have to guess when there is enough training data, if there is not, learning will fail and the system will have to be set up for collecting more training data.

Consider the set of multi-modal capable online learning methods for highdimensional inputs and continuous outputs, one property of this set stands out, its suprisingly low cardinality, at least among technical systems. The most successful learning systems of this type seem to exist in the biological world. The biological vision systems continue to provide inspiration and ideas for the design of their technical counterparts, however, biological vision systems do have weak spots where technical systems perform better. The main question still remains: what parts should we imitate and what parts should be designed differently? There is still much to explore in this area around the borders between computer vision, machine learning, psychology and neurophysiology.

\subsection{Outline Part I: Background Theory}

Following this introduction, the Perception-Action mapping is presented in chapter 2. The general objective is to learn perception-action mappings, enabling systems to operate autonomously. Three problems are explored: vision-based autonomous driving, inverse kinematics and control of a dynamic system.

Chapter 3 presents an overview of learning methods and different sources of training data. The relations to common classifications of learning methods are 

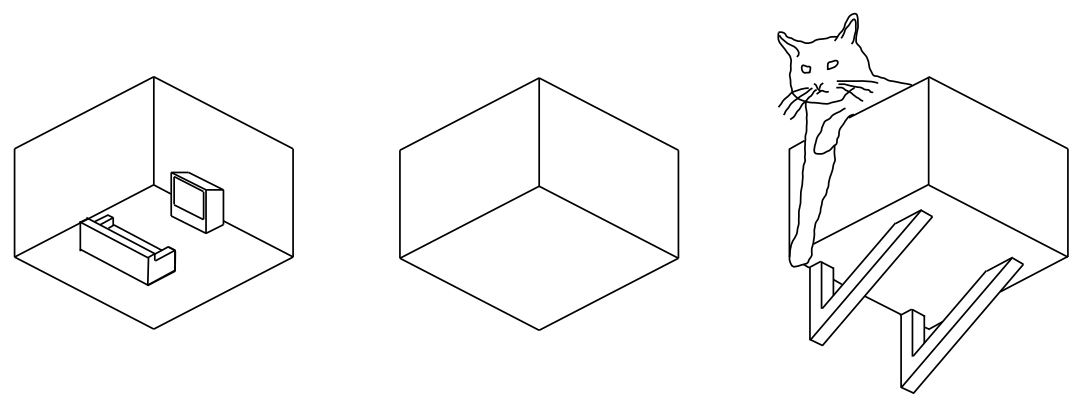

Figure 1.1: Illustration of the multi-modal interpretation of visual perception. There are at least two different interpretations of the middle figure. To the left and to the right, the same figure appears again, with some more visual clues for selecting one interpretation, one mode. A third interpretation is three flat parallelograms. There is a continous set of three dimensional figures, with more or less sharp corners, whose orthogonal projection would produce the middle figure. The reason why the options with right angle corners seem more commonly perceived is beyond the scope of this text. Illustration courtesy of Kristoffer Öfjäll, 2014.

explored. A selection of learning methods appearing in the included publications are presented in more detail.

Representations of inputs, outputs and the learned model are presented in chapter 4 . The chapter is primarily focused on collections of local and simple models, where the descriptive power originates from the relations of the local models.

In chapter 5, some highlights of the experiments in the included publications are presented. The opportunity is taken to elaborate on the differences and similarities between experiments from different publications. This material is not available in any of the publications alone. Finally, conclusions and some directions for future research are presented in chapter 6 .

\subsection{Outline Part II: Included Publications}

Preprint versions of four publications are included in Part II. The full details and abstracts of these papers, together with statements of the contributions made by the author, are summarized below.

\section{Paper A: Autonomous Navigation and Sign Detector Learning}

L. Ellis, N. Pugeault, K. Öfjäll, J. Hedborg, R. Bowden, and M. Felsberg. Autonomous navigation and sign detector learning. In Robot Vision (WORV), 2013 IEEE Workshop on, pages 144-151, Jan 2013. 


\begin{abstract}
:
This paper presents an autonomous robotic system that incorporates novel Computer Vision, Machine Learning and Data Mining algorithms in order to learn to navigate and discover important visual entities. This is achieved within a Learning from Demonstration (LfD) framework, where policies are derived from example state-to-action mappings. For autonomous navigation, a mapping is learnt from holistic image features (GIST) onto control parameters using Random Forest regression. Additionally, visual entities (road signs e.g. STOP sign) that are strongly associated to autonomously discovered modes of action (e.g. stopping behaviour) are discovered through a novel Percept-Action Mining methodology. The resulting sign detector is learnt without any supervision (no image labeling or bounding box annotations are used). The complete system is demonstrated on a fully autonomous robotic platform, featuring a single camera mounted on a standard remote control car. The robot carries a PC laptop, that performs all the processing on board and in real-time.
\end{abstract}

\title{
Contribution:
}

This work presents an integrated system with three main componets: learning visual navigation, learning traffic signs and corresponding actions, and, obstacle avoidance using monocular structure from motion. All processing is performed on board on a laptop. The author's main contributions includes integrating the systems on the intended platform and performing experiments, the latter in collaboration with Liam, Nicolas and Johan.

\section{Paper B: Online Learning of Vision-Based Robot Control during Au- tonomous Operation}

Kristoffer Öfjäll and Michael Felsberg. Online learning of vision-based robot control during autonomous operation. In Yu Sun, Aman Behal, and Chi-Kit Ronald Chung, editors, New Development in Robot Vision. Springer, Berlin, 2014.

\begin{abstract}
:
Online learning of vision-based robot control requires appropriate activation strategies during operation. In this chapter we present such a learning approach with applications to two areas of vision-based robot control. In the first setting, selfevaluation is possible for the learning system and the system autonomously switches to learning mode for producing the necessary training data by exploration. The other application is in a setting where external information is required for determining the correctness of an action. Therefore, an operator provides training data when required, leading to an automatic mode switch to online learning from demonstration. In experiments for the first setting, the system is able to autonomously learn the inverse kinematics of a robotic arm. We propose improvements producing more informative training data compared to random exploration. This reduces training time and limits learning to regions where the learnt mapping is used. The learnt region is extended autonomously on demand. In experiments for the second setting, we present an autonomous driving system learning a mapping from visual input to control signals, which is trained by manually steering
\end{abstract}


the robot. After the initial training period, the system seamlessly continues autonomously. Manual control can be taken back at any time for providing additional training.

\title{
Contribution:
}

This work presents two learning robotics systems where both learning and operation is online. The primary advantage compared to the system in paper $\mathrm{A}$ is the possibility to seamlessly switch to training mode if the initial training is insufficient. The author developed the ideas leading to this publication, implemented the systems, performed the experiments and did the main part of the writing.

\section{Paper C: Biologically Inspired Online Learning of Visual Autonomous Driving}

Kristoffer Öfjäll and Michael Felsberg. Biologically inspired online learning of visual autonomous driving. In Proceedings of the British Machine Vision Conference. BMVA Press, 2014.

\begin{abstract}
:
While autonomously driving systems accumulate more and more sensors as well as highly specialized visual features and engineered solutions, the human visual system provides evidence that visual input and simple low level image features are sufficient for successful driving. In this paper we propose extensions (nonlinear update and coherence weighting) to one of the simplest biologically inspired learning schemes (Hebbian learning). We show that this is sufficient for online learning of visual autonomous driving, where the system learns to directly map low level image features to control signals. After the initial training period, the system seamlessly continues autonomously. This extended Hebbian algorithm, qHebb, has constant bounds on time and memory complexity for training and evaluation, independent of the number of training samples presented to the system. Further, the proposed algorithm compares favorably to state of the art engineered batch learning algorithms.
\end{abstract}

\section{Contribution:}

This work presents a novel online multimodal Hebbian associative learning scheme which retain properties of previous associative learning methods while improving performance such that the proposed method compares favourably to state of the art batch learning methods. The author developed the ideas and the extensions of Hebbian learning leading to this publication, implemented the demonstrator system, performed the experiments and did the main part of the writing.

\section{Paper D: Combining Vision, Machine Learning and Automatic Control to Play the Labyrinth Game}

Kristoffer Öfjäll and Michael Felsberg. Combining vision, machine learning and automatic control to play the labyrinth game. In Proceedings of SSBA, Swedish Symposium on Image Analysis, Feb 2012.

\section{Abstract:}

The labyrinth game is a simple yet challenging platform, not only for humans 
but also for control algorithms and systems. The game is easy to understand but still very hard to master. From a system point of view, the ball behavior is in general easy to model but close to the obstacles there are severe non-linearities. Additionally, the far from flat surface on which the ball rolls provides for changing dynamics depending on the ball position.

The general dynamics of the system can easily be handled by traditional automatic control methods. Taking the obstacles and uneven surface into account would require very detailed models of the system. A simple deterministic control algorithm is combined with a learning control method. The simple control method provides initial training data. As the learning method is trained, the system can learn from the results of its own actions and the performance improves well beyond the performance of the initial controller.

A vision system and image analysis is used to estimate the ball position while a combination of a PID controller and a learning controller based on LWPR is used to learn to steer the ball through the maze.

\section{Contribution:}

This work presents an evaluation system for control algorithms. A novel learning controller based on LWPR is evaluated and it is shown that the performance of the learning controller can improve beyond the performance of the teacher. The author initiated and developed the ideas leading to this publication, implemented the demonstrator system, performed the experiments and did the main part of the writing.

\section{Other Publications}

The following publications by the author are related to the included papers.

Kristoffer Öfjäll and Michael Felsberg. Rapid explorative direct inverse kinematics learning of relevant locations for active vision. In Robot Vision (WORV), 2013 IEEE Workshop on, pages 14-19, Jan 2013.

Kristoffer Öfjäll and Michael Felsberg. Online learning and mode switching for autonomous driving from demonstration. In Proceedings of SSBA, Swedish Symposium on Image Analysis, March 2014.

Kristoffer Öfjäll and Michael Felsberg. Integrating learning and optimization for active vision inverse kinematics. In Proceedings of $S S B A$, Swedish Symposium on Image Analysis, March 2013. 


\section{Chapter 2}

\section{Perception-Action Mappings}

For many systems, both biological and technical, a satisfactory mapping from perceptions to actions is essential for survival or successful operation within the intended application. There exist a wide range of these types of mappings. Some are temporally very direct such as reflexes in animals and obstacle avoidance in robotic lawn mowers. Others depend on previous perceptions and actions such as visual flight stabilization in insects $[48,9]$ and technical systems for controlling dynamical processes in general, one example is autonomous helicopter aerobatics [1]. Further, there are systems where actions depend on several different perceptions more or less distant in time, systems featuring such things as memory and learning in some sense. Such systems can work through a mechanism where certain perceptions alter the perception-action mappings of other perceptions. This will be further discussed in chapter 3 .

This work primarily regards technical systems, where the perceptions are of visual nature. Three different systems will be studied, a system for autonomous driving, a system for robotic arm control and a system for controlling a dynamic system.

\subsection{Vision Based Autonomous Driving}

Autonomously driving vehicles are gaining poplarity. One just needs to consider the latest DARPA Grand Challenge. Looking at these cars there is one thing that stands out, the abundance of sensors. Many of which are active, that is, the sensors emit some type of signal wich interact with the environment and some parts of the signal return to the sensor. This includes popular sensors such as radars, sonars, laser scanners and active infra-red cameras, with an infra-red light source contained onboard the vehicle. In an environment with increasing number of autonomous vehicles, these active sensors may interfer with sensors of another vehicle. Fig. 2.2 illustrates an, admittedly slightly exaggerated, block diagram of this type of conventional autonomous vehicle.

On the other hand, all these sensor modalities are not necessary for driving. A 


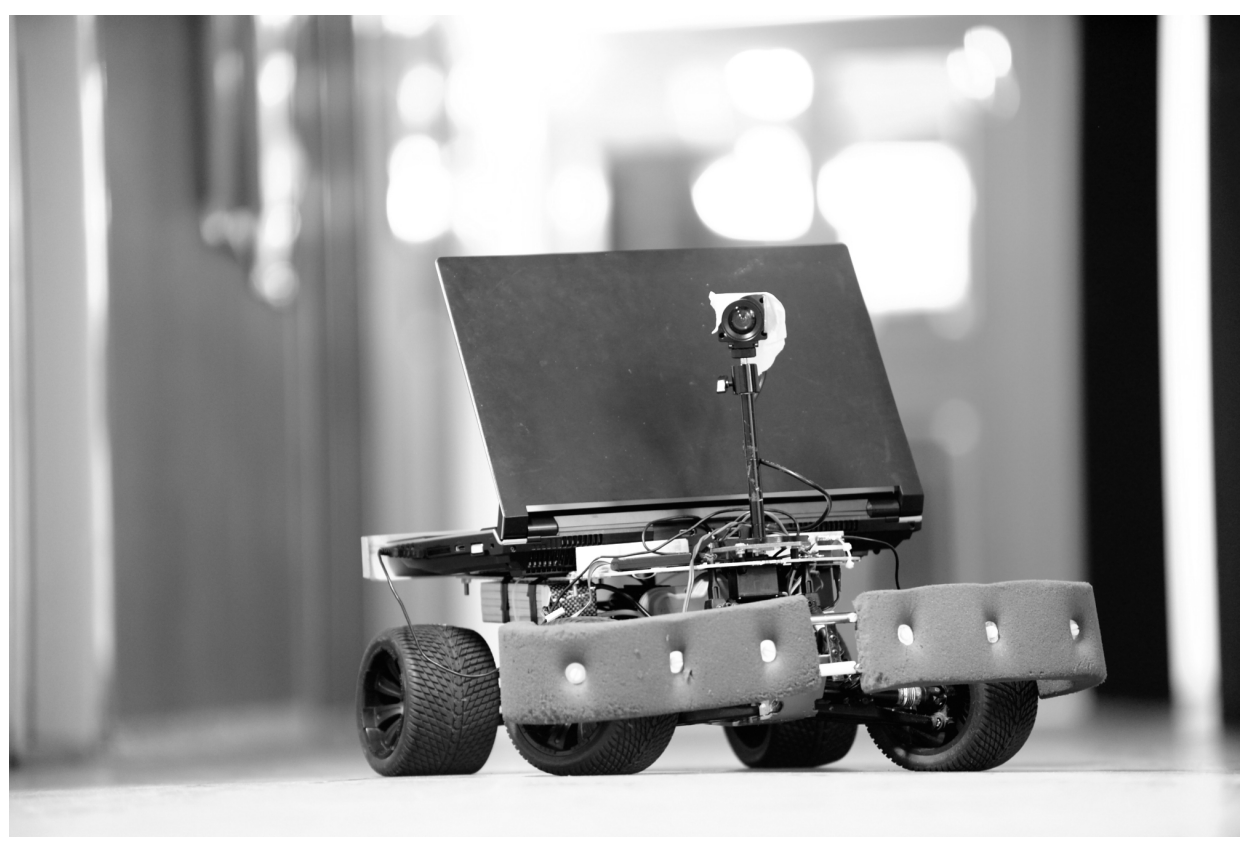

Figure 2.1: System for visual autonomous driving experiments.

human operator can successfully drive a car using only visual input. Any remotely controlled toy car with a video link is an experiment confirming this. Our approach is to remove all unneccessary components and directly map visual perceptions to control actions, Fig 2.3.

The hardware platform itself is shown in Fig. 2.1, a standard radio controlled car with a laptop for onboard computing capabilities. The car is equipped with a camera and hardware transferring control commands from the onboard computer to the car. The control signals from the original transmitter are rerouted to the onboard computer, allowing a human operator to demonstrate correct driving behavior.

The action space of this system is a continous steering signal. The perception space contains the images captured by the onboard camera. Currently, the driving speed is constant. The vehicle operates at walking speed and thus the dynamics of the vehicle is negligible. The rate of turn is approximately only dependet on the last steering command, not previous actions. 


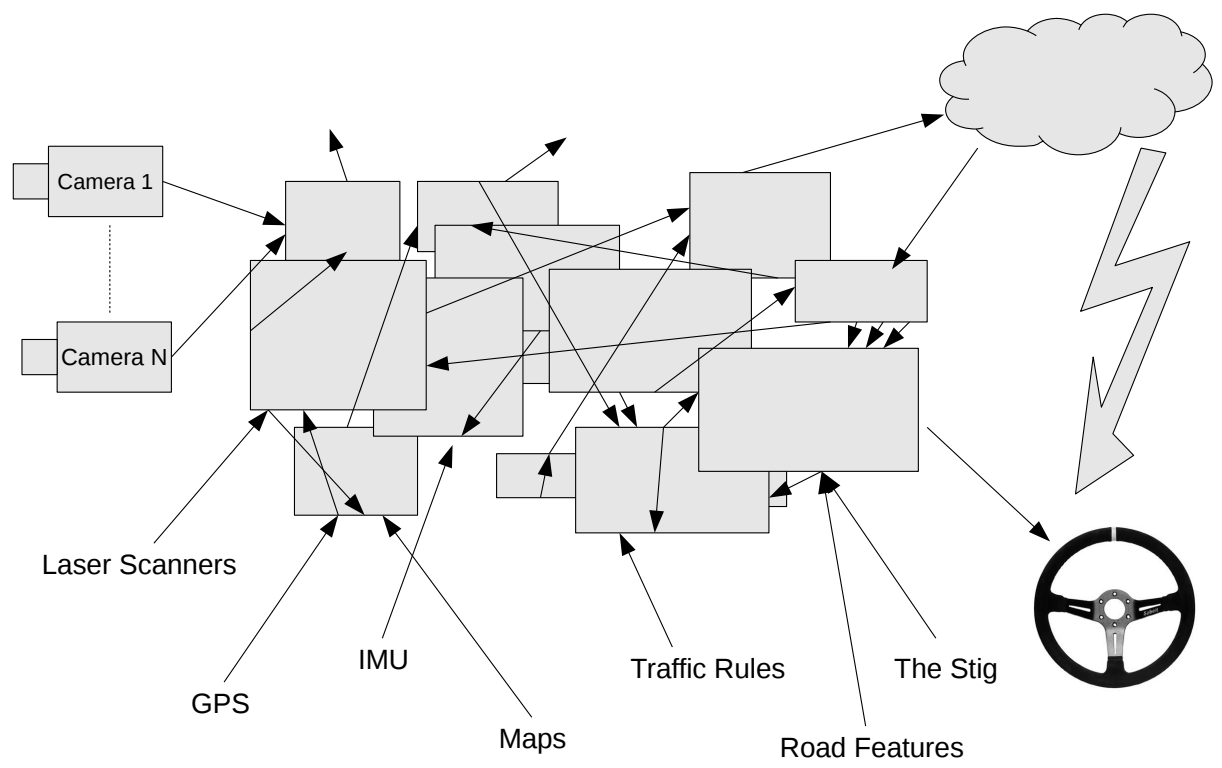

Figure 2.2: Common approach to autonomous driving.

\section{$2.2 \quad$ Inverse Kinematics}

The inverse kinematics of a robotic arm is a mapping from a desired pose of the end effector to the angle of each joint, that is, given a desired position and orientation of the end effector, the inverse kinematics should generate a joint angle for each joint of the robot such that the desired pose is attained. The forward kinematics is the opposite task, given the joint angles, predict the position and orientation of the end effector.

For a serial manipulator, each link of the arm is attached to previous link in a serial fashion from the robot base to the end effector. In such a case, calculating the forward kinematics reduces to a series of rotations and translations relating the base coordinate system to the end effector coordinate system. However, the forward kinematics function is not necessarily injective, different joint configurations may result in the same end effector pose. In such a case, the inverse kinematics problem has several different solutions.

There are also configurations of the arm known as singularities, where the rotational axes of two joints coincide. In such a case, the section of the arm between these two joints may be arbitrarily rotated. The issue occurs when the robot is required to attain a pose infinitesimally close to the singularity which requires a particular configuration of the middle section, typically the correct orientation of 


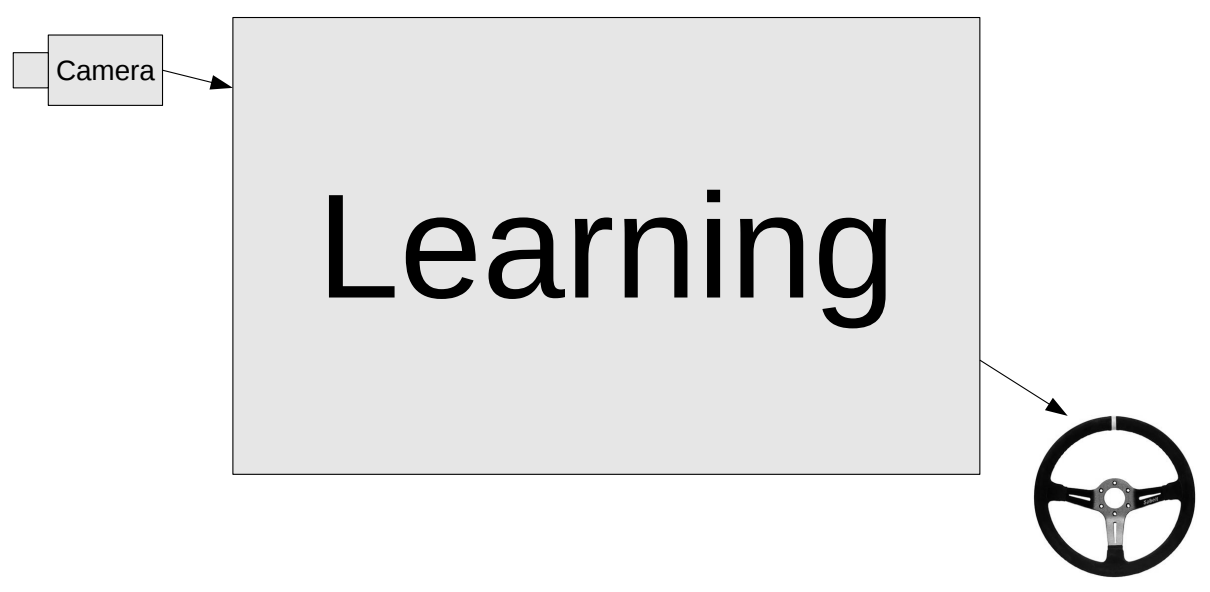

Figure 2.3: Our approach to autonomous driving.

a rotation axis of a joint between the two coinciding joints. For a constant speed pose trajectory, this may require an infinite rotational speed of the middle section.

For a redundant robotic arm, the dimensionality of the joint space is larger than the dimensionality of the pose space. This is the case for the robot in Fig. 2.4, which has seven rotational joints. Given a desired pose, the solutions lie in a, possibly not connected, one dimensional subset of the joint space. Here the action space is the joint configuration space and the perception space is the space of end effector poses.

\subsection{Dynamic System Control}

The dynamical system in question is the Brio labyrinth game. Using the game for evaluation of perception-action systems brings some advantages. Primarily the labyrinth is recognized by a large group of people which can directly relate to the level of the challenge. Theoretically, the motion of the ball is rather simple to describe as long as the ball does not interact with the obstacles in the maze. However, imperfections during manufacturing introduces non-linearities.

The goal is to successfully guide the ball through the maze while avoiding holes. The action space is the two dimensional space of tilt angles of the maze. The 


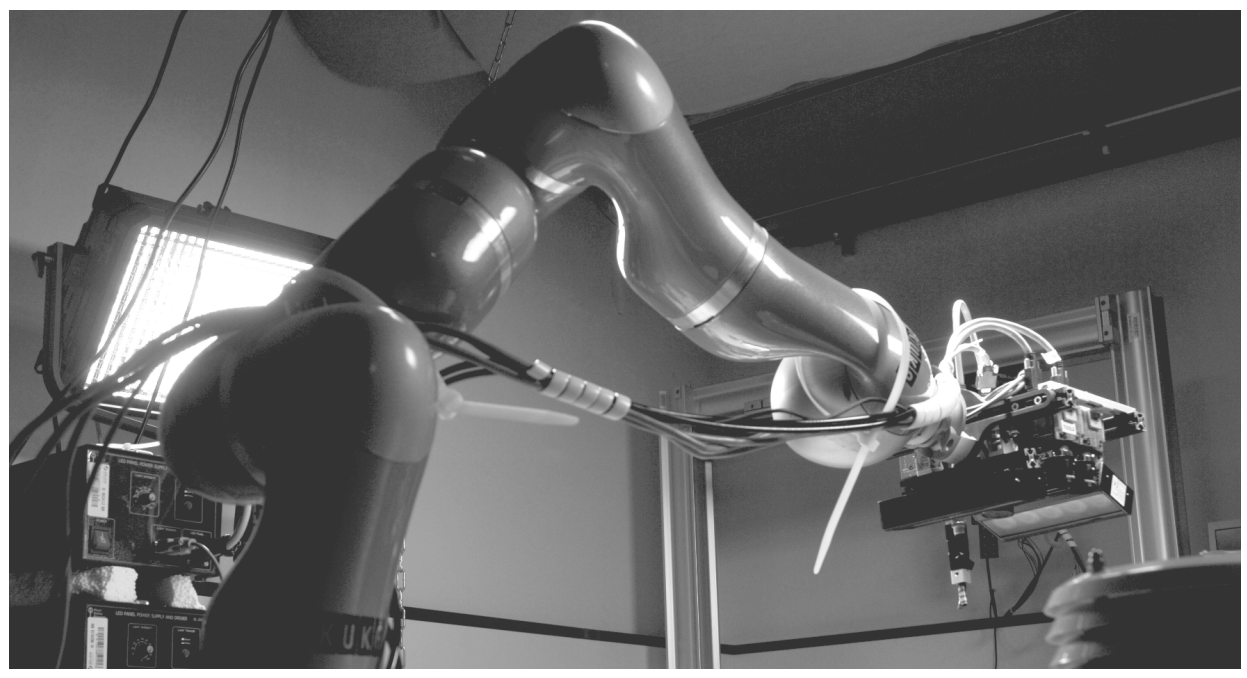

Figure 2.4: Robot setup for active visual inspection.

perception space is the game itself, captured by a camera. The current platform contains deterministic routines for extraction of the ball position in the maze, as well as for estimation of ball velocity.

In the current experimental setup, the desired path is provided to the system in advance, such that the system only needs to learn how to control the ball. An alternative setup would be not to provide the system with the desired path or even the objective of the game for that matter. This alternative setup is similar to the autonomous driving task in the sense that also the objective of the game would have to be learned, possibly from demonstration. 


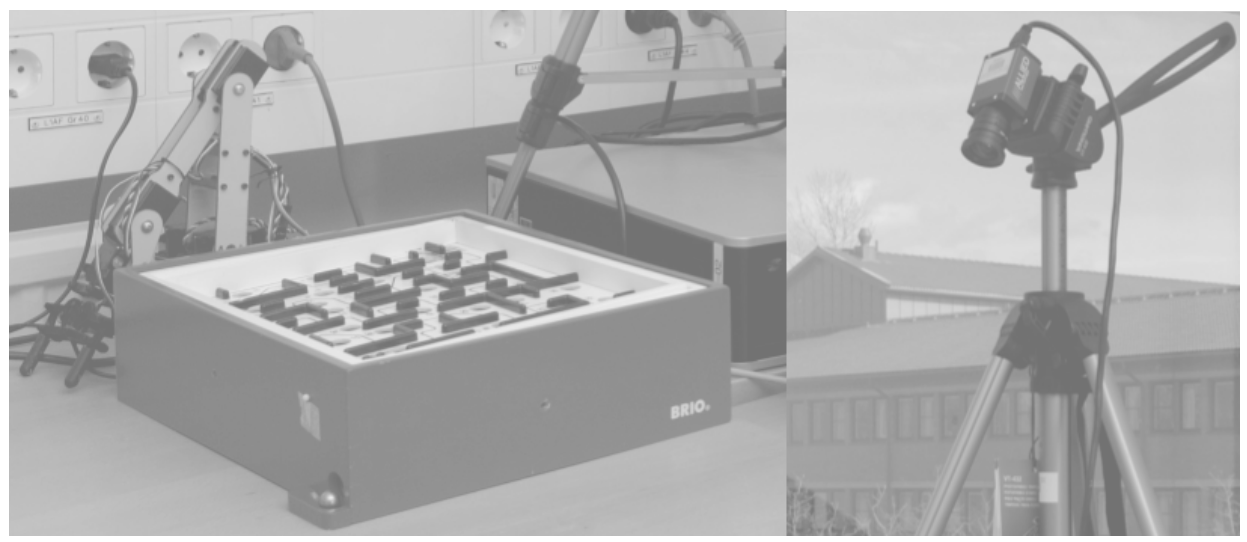

Figure 2.5: Dynamical labyrinth game system. 


\section{Chapter 3}

\section{Learning Methods}

There are several ways of obtaining perception-action mappings as mentioned in chapter 2. For the simpler tasks, it is possible to formulate an explicit mathematical expression generating successful actions depending on the perceptions. One such example is using a PID-controller for the labyrinth game [28]. However, that approach does not account of position dependent changes in ball behavior, such as obstacles and deformations of the maze. Further, the static expression cannot adapt to changes in the controlled system over time.

For biological systems, learning is common on several levels and has been extensively studied. One well known example of learning on individual level are the experiments on conditioning in dogs by Pavlov [36]. Pavlov noted that the salivation of the dogs increased when they were presented with food. By repeatedly and consequently combining the presentation of food with another stimuli unrelated to food, the dogs related the earlier unrelated stimuli to food. After some training, the new stimuli was sufficient for generating increased salivation.

There are also processes which could be considered learning on the level of species, where common traits in a group of animals adapt to the environment by means of replacing individuals but where the individual animals do not necessarily change. Darwin [11] provided many examples where this type of learning probably had taken place. The natural variation which Darwin refers to, where random changes appear in individual animals, can be compared to random exploration for artificial learning systems.

Most of the early studies on biological systems were, from a learning perspective, more concerned about that there was learning, not how this learning would come about. For building artificial learning systems, the latter would be more rewarding in terms of providing implementational ideas. One idea of learning in a biological neural network was proposed by Donald Hebb [21]. Simplified, the idea was that neurons often simoultanously activated tend to get stronger connections. This made its way to artificial learning systems and is referred to as Hebbian learning.

Also the ideas from Darwin made their way to the technical systems, some in the form of genetic algorithms where individuals represent different parameter 
settings and these individuals compete and recombine. This was used to find the parameters and structure of an Othello playing neural network [27].

In section 3.1, properties of learning systems which are fundamental to the presentation in the included publications are presented. In section 3.2, the set of learning systems is partitioned depending on the nature of training information available to the system. This is closely related to the more common partitioning depending on the systems themselves such as into supervised learning, reinforcement learning and unsupervised learning. The final sections present a selection of learning systems appearing in the included publications.

\subsection{Classification of Learning Methods}

There are several ways of classifying learning methods. In this section a set of properties of learning systems will be presented. The categories in this section are not mutually exclusive, a particular learning system may possess several of the presented properties.

\subsubsection{Online Learning}

The purpose of online learning methods is the ability to incorporate new training data without retraining the full system [39]. However, there is a lack of consesus in literature regarding explicit demands for a learning system to be classified as online. Here online systems will be required to fulfill a set of requirements, both during training and prediction:

- The method should be incremental, that is, the system should be able to incorporate a new training sample without access to previous or later training samples.

- The computational demand of incorporating a new training sample or making a single prediction should be bounded by a finite bound, especially, the bound shall be independent of the number of training samples already presented to the system.

- The memory requirement of the internal model should be bounded by a finite bound, especially, the bound shall be independent of the number of training samples presented to the system.

The first requirement is common to all definitions of online learning systems encountered so far. The last two are not always present, or less strict, such as allowing a logarithmic increase in complexity with more training data. For a strict treatment, it may be necessary to let the bounds depend on the complexity of the underlying model to be learned.

\subsubsection{Active Learning}

With active learning, the learning algorithm can affect the generation or selection of training data in some way. Also in this case there is a lack of consensus in 
literature, however, there is a survey [46] illuminating different aspects of active learning. In this case, active learning is applicable to inverse kinematics learning and the labyrinth game where training data can be generated by exploration. In contrast, for the autonomous driving system, the demonstrator selects the training set, wich cannot be affected by the car.

There is also random exploration, also known as motor babbling. During random exploration, randomly selected actions are performed, hopefully generating informative training data. With a more active approach, exploiting already learnt connections increases the efficiency of the exploration $[40,2,8]$.

\subsubsection{Multi Modal Learning}

Here multi modal will express the ability of the learning system to learn general mappings which are not functions in a mathematical sense. The name stems from the output of such systems possibly having multiple modes. This is also referred to as multiple hypotheses. In other contexts, multi modal is used to denote properties of the input or output spaces consisting of multiple modes such as vision and audio sensors combined.

A multi modal mapping is a one-to-many or many-to-many mapping. It is most easily described in terms of what it is not. A unimodal mapping is a many-to-one mapping, a function in mathematical terms, or a one-to-one mapping, an injective function. For a unimodal perception-action mapping, each perception maps to precisely one action, but in general, different perceptions may map to the same action. For an injective perception-action mapping, two different perceptions will not map to the same action.

For a multi modal perception-action mapping, each perception may map to several different actions. This type of mapping cannot be described as a function. Consider a car at a four-way intersection. While the visual percept is equal in all cases, the car may turn left, turn right or continue straight. The same perception is mapped to three different actions, where the specific action only depends on the intention of the driver.

Most learning methods are only designed for, and can only learn, unimodal mappings [14]. This is illustrated in figure 3.1 where samples from a multimodal mapping (crosses) are shown. Each input perception maps to either of two output actions. An input of 0.5 maps either to output 0.25 or output 0.5 . Five learning systems are trained using these samples and the outputs generated by the system in response to inputs between 0 and 1 are plotted in the figure. These systems are linear regression, Support Vector Regression (SVR) [6], Realtime Overlapping Gaussian Expert Regression (ROGER) [19], Random Forest Regression (RFR) [25] and qHebb [31].

The unimodal methods (linear regression and RFR) tend to generate averages between the two modes (linear regression) or discontinous jumps between the modes (RFR). The multi-modal capable methods (SVR, ROGER and qHebb) selects the stronger mode in each region. Support vector regression is however a batch learning method. ROGER is slightly affected by the weaker mode and slows 


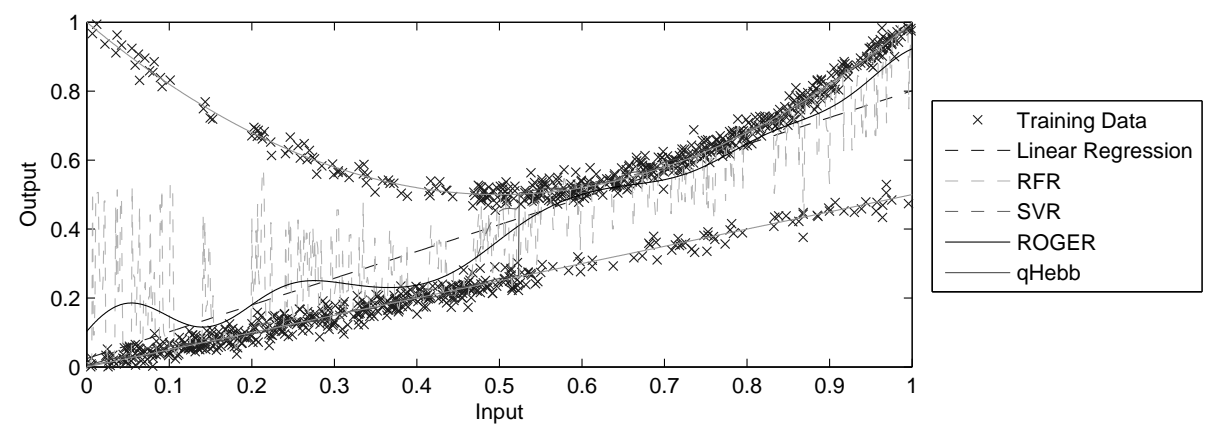

Figure 3.1: qHebb, ROGER, SVR, RFR and linear regression on a synthetic multimodal dataset. During evaluation, the learning methods should choose the stronger of the two modes present in the training data. Unimodal methods tend to mix the two modes.

down with increasing number of training samples. In general, there is a shortage of fully online multi-modal learning methods.

\subsection{Training Data Source}

For a system to learn a perception-action mapping, training data have to be generated by, or be made available to, the system. The possible sources of training data depend on the system and its possibilities of self-evaluation. For the labyrinth game and the inverse kinematics, an executed action will result in a new position and velocity of the ball or a certain pose of the end effector. In both cases, the new perception can be compared to the desired ball trajectory or the desired pose and the successfulness of the action can be determined. For these systems, it is possible to explore the available action space to find appropriate actions for certain perceptions.

On the other hand, for the autonomos driving system, the correctness of an action depends on the local road layout and driving conventions, neither available to the system. Thus, the system cannot evaluate its own actions and explorative learning is not possible.

\subsubsection{Explorative Learning}

Explorative learning, or self-supervised learning, is possible when the system is able to assess the success of its own actions. There are also examples where a robot has learned to do visual tracking with a camera and can use this information to learn to follow objects using a laser scanner [10].

Self-supervised learning differ from unsupervised learning [20] in that unsupervised learning usually concerns dimensionality reduction, finding structure in the input data. There is no output or action to be predicted. The output from an 
unsupervised learning system is a new, often lower dimensional, representation of the input space.

Explorative learning may be either fully random, as in motor babbling, fully deterministic as when using gradient descent [30], or any combination thereof like directed motor babbling [40]. A fully random exploration method may not produce useful training data, or as described by Schaal: falling down might not tell us much about the forces needed in walking [42]. A fully deterministic approach will on the other hand make the same prediction in the same situations, thus possibly better solutions may be missed.

\subsubsection{Learning from Demonstration}

Learning from demonstration is one variant of supervised learning. Training data are the perceptions (inputs) and the corresponding actions (outputs) encountered while the system is operated by another operator, typically a human. The operator provides a more or less correct solution, however, it may be a sub-optimal solution, it may be disturbed by noise and it may not be the only solution. One example of multiple solutions are the multimodal mappings previously examplified by a road intersection.

In the autonomous driving case, it is not possible for the system to judge the correctness of a single action. It is only by the distribution of actions demonstrated for similar perceptions something may be said regarding the correct solution or solutions. Hopefully the demonstrator is unbiased such that for each mode, the mean action is correct. A human operator may steer a bit to little around one corner and a bit to much in another corner. The autonomous driving system thus adopts the driving style from the demonstrator, such as a tendency to cut corners.

Learning from demonstration is also available in the labyrinth game, here the system is able to judge the demonstrated actions in terms of how these actions alter the ball trajectory with respect to the desired trajectory. For the labyrint, demonstrating bad actions will not have negative impact on the performance of the learning system while even short sequences of good actions will facilitate faster learning, see section 5.3.

Learning from demonstration tends to generate training data with certain properties which some learning methods may be suceptible to. First, the training data is typically temporally correlated. For autonomous driving, there may be several frames of straight road before the first example of a corner is produced. For online learning algorithms operating on batch data, a random permutation of the training data has shown to produce better learning results [20]. This is not possible in a true online learning setting.

Second, the training data is biased. While there is a vast amount of training data available from common situtations, there may only be a few examples of rare situations. However, these rare situations are at least equally important as the regular situations for successful autonomous driving. Thus, the relative occurance of certain perceptions and actions in the training data does not reflect their importance. For ALVINN, special algorithms had to be developed to reduce the training set bias by removing common training data before the learning system [3]. 
After discussions with other researchers within the autonomous vehicle community it is clear that batch learning methods suffer from a common issue in training data. Usually, the training data only contain examples of the vehicle driving successfully on the road. However, if the vehicle deviates slightly from the correct path during autonomous operation, there are no training examples demonstrating driving back onto the correct path. This is an advantage for online learning methods as if, during autonomous operation, the vehicle deviates from the correct path, manual control can be reaquired and a corrective maneuver can be carried out, while at the same time providing the learning system with training data demonstrating this correction [31].

\subsubsection{Reinforcement Learning}

Reinforcement learning [20] is a special type of learning scenario where the teacher only provides performance feedback to the learning system, not full input-output examples as for the learning from demonstration case. Learning is in general slower as the learning system may have to try several actions before making progress. After a solution is found, there is a trade-off between using the found solution and searching for a better solution, known as the exploration-exploitation dilemma. One possible scenario of reinforcement learning for autonomous navigation is a teacher providing feedback on how far from the desired path the vehicle is going. This possibility has not been explored in any of the included publications.

\subsection{Locally Weighted Projection Regression}

Locally weighted projection regression [50], LWPR, is a unimodal online learning method developed for applications where the output is dependent on low dimensional inputs embedded in a high dimensional space. However there are issues with image feature spaces with thousands of dimensions or more. LWPR is an extension of locally weighted regression [43]. The general idea is to use the output from several local linear models weighted together to form the output.

The output $y_{d k}$ for each local model $k$ for dimension $d$ consists of $r_{k}$ linear regressors

$$
y_{d k}=\beta_{d k}^{0}+\sum_{i=1}^{r_{k}} \beta_{d k i} \mathbf{u}_{d k i}^{T}\left(\mathbf{x}_{d k i}-\mathbf{x}_{d k}^{0}\right)
$$

along different directions $\mathbf{u}_{d k i}$ in the input space. Each projection direction and corresponding regression parameter $\beta_{d k i}$ and bias $\beta_{d k}^{0}$ are adjusted online using partial least squares. Variations in the input explained by each regression $i$ is removed from the input $\mathbf{x}$ generating the input to the next regressor $\mathbf{x}_{d k(i+1)}$.

The total prediction $\hat{y}_{d}$ in one output dimension $d$

$$
\hat{y}_{d}=\frac{\sum_{k=1}^{K} w_{d k} y_{d k}}{\sum_{k=1}^{K} w_{d k}}
$$

depends on the distance from the center $\mathbf{c}_{d k}$ of each of the local models. Normally 
a Gaussian kernel is used, generating the weights

$$
w_{d k}=\exp \left(-\frac{1}{2}\left(\mathbf{x}-\mathbf{c}_{d k}\right)^{T} \mathbf{D}_{d k}\left(\mathbf{x}-\mathbf{c}_{d k}\right)\right)
$$

where the metric $\mathbf{D}_{d k}$ is updated using stochastic gradient descent on the prediction error of each new training data point. The model centers $\mathbf{c}_{d k}$ remain constant. New models are created when the weights of all previous models for a new training sample is below a fixed threshold, the new model is centered on the new training sample. The distance matrix for the new model is intialized to a fixed matrix, which is a user selectable parameter of the method.

\subsubsection{High Dimensional Issues}

Although the input is projected onto a few dimensions, the distances for the weights still live in the full input space. The online property of the method depends on convergence to a finite number of local models and of a limited number of projection directions within each model. In the experiment presented in [50], a one dimensional output was predicted from a 50 dimensional space where the output depended on a two dimensional subspace.

Using full 2048 dimensional feature vectors as input, each local model required some hundred megabytes of primary memory for the autonomous vehicle. Increasing the size of the initial local models by setting smaller entries in the initial $\mathbf{D}$ parameter reduced the problem, however, for longer training times the dimensionality of the input space had to be reduced before using LWPR [33]. Further, the method is unimodal such that actions corresponding to the same perception are averaged. This is an issue when the autonomous vehicle encounters an obstacle straight ahead and the training data contains examples of evasive maneuvers both to the left and to the right.

\subsection{Random Forest Regression}

A forest [5] is a collection of decision trees where the output of the forest is taken to be the average over all trees. A decision tree is a, usually binary, tree where each inner node contains a test on input data. The test decides a path through the tree. Each leaf contains either a class label for classification trees or a regression model for regression trees. As the model in each leaf only has to be valid for input data related to that leaf, the model can in general be significantly simpler than a model supposed to be valid in the full domain. In [13], a model of order zero was used, the mean value of all training data ending up in the leaf in question.

There is a large collection of approaches for building trees and selecting split criteria [41]. For a collection of trees, a forest, Breiman noted that best performance was obtained for uncorrelated trees, however, building several trees from the same training data tend to generate dependencies between the trees.

In 1996 bagging was proposed an attempt to reduce inter-tree dependecies [4]. The idea is to use a random subset of the training data to build each tree in the 
forest. Later this was generalized as random forests where a random parameter vector $\Theta_{k}$ is generated for each tree $k$ and governs its construction [5]. All $\Theta_{k}$ are independet and identically distributed. For bagging, $\Theta_{k}$ is a random vector with binary entries and as many elements as there are training data entries. Each element in $\Theta_{k}$ determines if the corresponding traing sample is to be used for building tree $k$. Also split criteria and thresholds may be randomly selected, usually after normalizing the input data by removing the mean and scaling by the inverse standard deviation in each dimension [5].

In the original formulation, the output from the whole forest was taken as the mean output of all trees in the forest. Later, using the median was proposed [38], which was shown to increase regression accuracy for regression onto steering control signals [13]. Using the mean over the forest tended to generate under-estimated steering predictions.

Further, the random forests described so far are not able to handle multimodal outputs. This is seen in figure 3.1 where the prediction from the random forest regressor jumps chaotically between the two output modes present in the training data. However, extending the trees with multi-modal capable models in the leaves and making suitable changes to the split criteria selection, it is possible to construct random forests which properly handle multi-modal outputs.

\subsection{Hebbian Learning}

The name Hebbian originates from the Canadian psychologist Donald Olding Hebb. In his 1949 book he proposed a mechanism by which learning can come about in biological neural networks [21]. The often quoted lines, referred to as Hebbs rule, read:

Let us assume then that the persistence or repetition of a reverberatory activity (or "trace") tends to induce lasting cellular changes that add to its stability. The assumption can be precisely stated as follows: When an axion of cell $\mathrm{A}$ is near enough to excite a cell B and repeatedly or persistently takes part in firing it, some growth process or metabolic change takes place in one or both cells such that A's efficiency, as one of the cells firing B, is increased.

- Donald Hebb, 1949 [21]

Simplified and applied to terms of perception and action, this would imply that for any perception repeatedly present simoultanously with a perticular action, the particular action will more and more easily be triggerd by the presense of this particular perception. This relates to the dogs of Pavlov, whose salivation action was possible to trigger with perceptions not related to food.

For a technical system, one of the simplest examples of Hebbian learning is a scalar valued linear function of a vector $\mathbf{x}$ parameterized by a weight vector $\mathbf{w}$ with synaptic strengths,

$$
y=\mathbf{w}^{\mathrm{T}} \mathbf{x}
$$


Introducing a discrete time parameter $t$ and a set of training data $\left(\mathbf{x}_{1}, \mathbf{x}_{2}, \ldots\right)$, a simple application of Hebbs rule generates the synaptic weight update scheme (Equation (8.37) in [20])

$$
\mathbf{w}_{t+1}=\mathbf{w}_{t}+\eta y_{t} \mathbf{x}_{t}
$$

where $\eta$ sets the learning rate.

Direct application of the learning rule (3.5) would lead to unlimited growth of the elements in the weight vector. This can be mitigated by introducing a normalization in each step

$$
\mathbf{w}_{t+1}=\frac{\mathbf{w}_{t}+\eta y_{t} \mathbf{x}_{t}}{\sqrt{\left(\mathbf{w}_{t}+\eta y_{t} \mathbf{x}_{t}\right)^{\mathrm{T}}\left(\mathbf{w}_{t}+\eta y_{t} \mathbf{x}_{t}\right)}}
$$

which, assuming a small $\eta$, can be simplified to

$$
\mathbf{w}_{t+1}=\mathbf{w}_{t}+\eta y_{t}\left(\mathbf{x}_{t}-y_{t} \mathbf{w}_{t}\right)
$$

This relation, known as Ojas rule, was shown by Oja [34] to converge to the largest principal component of $\mathbf{x}$, that is $\mathbf{w}$ converges to the eigenvector corresponding to the largest eigenvalue of the correlation matrix $\mathrm{E}\left[\mathbf{x x}^{\mathrm{T}}\right]$ under the assumptions of zero mean distribution of $\mathbf{x}$ and the existance of a uniqe largest eigenvalue. By removing the projections of the input vectors from the input vectors and learning a new $\mathbf{w}$, the second principal component can be obtained, and so forth.

The above example is unsupervised learning, the outcome does only depend on the input. There is no prediction of any output. A similar approach can be used for linear regression. Assume a linearly generated output $y$ depending on the input $\mathbf{x}$ and the fixed parameters in the vector $\beta$,

$$
y=\beta^{\mathrm{T}} \mathbf{x} \quad .
$$

Let $\left(y_{i}, \mathbf{x}_{i}\right)$ be training data pairs fulfilling $y_{i}=\beta^{\mathrm{T}} \mathbf{x}_{i}$ for $i=1,2, \ldots, N$. Further, let

$$
\mathbf{w}=\frac{1}{N} \sum_{i=1}^{N} \mathbf{x}_{i} y_{i}=\frac{1}{N} \sum_{i=1}^{N} \mathbf{x}_{i}\left(\beta^{\mathrm{T}} \mathbf{x}_{i}\right)
$$

that is, $\mathbf{w}$ is a weighted sum of the $\mathbf{x}_{i}$, where each term lies in the half-space $\left\{\mathbf{z}: \beta^{\mathrm{T}} \mathbf{z} \geq 0\right\}$. Given certain symmetry conditions on the distribution of the $\mathbf{x}_{i}$, it is geometrically clear that $\mathbf{w}$ will tend to be parallel to $\beta$ for increasing $N$ since the total contribution from the $\mathbf{x}_{i}$ orthogonal to $\beta$ will remain small compared to the total contributions along $\beta$. This is as each $\mathbf{x}_{i}\left(\beta^{\mathrm{T}} \mathbf{x}_{i}\right)$ will contribute a step in the positive $\beta$ direction with probability one ${ }^{1}$ while the total contribution orthogonal to $\beta$ is a random walk.

However, convergence is rather slow and the symmetry requirements on $\mathbf{x}_{i}$ limits the applicability of this direct Hebbian regression method. Convergence is illustrated in figure 3.2 for a $5 \mathrm{D}$ input space. For comparison, since there is no noise, the true parameter vector could be found from five linearly independet training samples by solving a linear equation system. From Hebbs book it is

\footnotetext{
${ }^{1}$ Assuming no impulse in the distribution at $\mathbf{x}_{i}=\mathbf{0}$.
} 
also clear that the proposed learning mechanism is not supposed to be applied directly to the values of entities to be learned, but to some other representation of the entities where several neurons are used to represent each entity. Such a representation is presented in chapter 4 .

Similar approaches exist in literature with theoretically more well founded analyzes of properties. Examples are Canonical Correlation Analysis (CCA) and Independent Component Analysis (ICA) [20].

\subsection{Associative Learning}

In associative learning, actions $\mathbf{y}$ are associated to perceptions $\mathbf{x}$. Here we will consider linear association such that actions and perceptions can be related by a linkage matrix $\mathbf{C}$,

$$
\mathbf{y}=\mathbf{C x} \quad .
$$

The elements of $\mathbf{x}$ and $\mathbf{y}$ usually represent the grade of activation of perceptions and actions and are thus non-negative.

Given a set of training examples $\left(\mathbf{x}_{i}, \mathbf{y}_{i}\right), i=1,2, \ldots, N$, a Hebbian approach for learning $\mathbf{C}$ is the sum of outer products

$$
\mathbf{C}=\sum_{i=1}^{N} \mathbf{y}_{i} \mathbf{x}_{i}^{\mathrm{T}}
$$

since for each outer product, each element in the matrix is large if both the corresponding perception and action are activated in the current training sample. Considering partial sums $\mathbf{C}_{i}=\mathbf{C}_{i-1}+\mathbf{y}_{i} \mathbf{x}_{i}^{\mathrm{T}}$, (3.11) is obviosly an online method. However, there are issues such as elements in $\mathbf{C}$ growing without bound. These are addressed in paper $\mathrm{C}$ [31].

The linear associative mapping (3.10) can be expressed as a single layer artificial neural network with linear activation functions where the synaptic weights are the elements in C, Fig. 3.3. In this simplest form, only linear relations can be learned (see the regression example in section 3.5). For learning more complex mappings, the approach of artificial neural networks and associative learning differ. Artificial neural networks employs non-linear activation functions and more involved structure with multiple neuron layers and possibly recurrent links. For associative learning, the linear mapping (3.10) is used but the representation of perceptions and actions is enhanced such that linear mappings on the new representation correspond to non-linear mappings in the original perception-action space. Representation is the matter of chapter 4. 

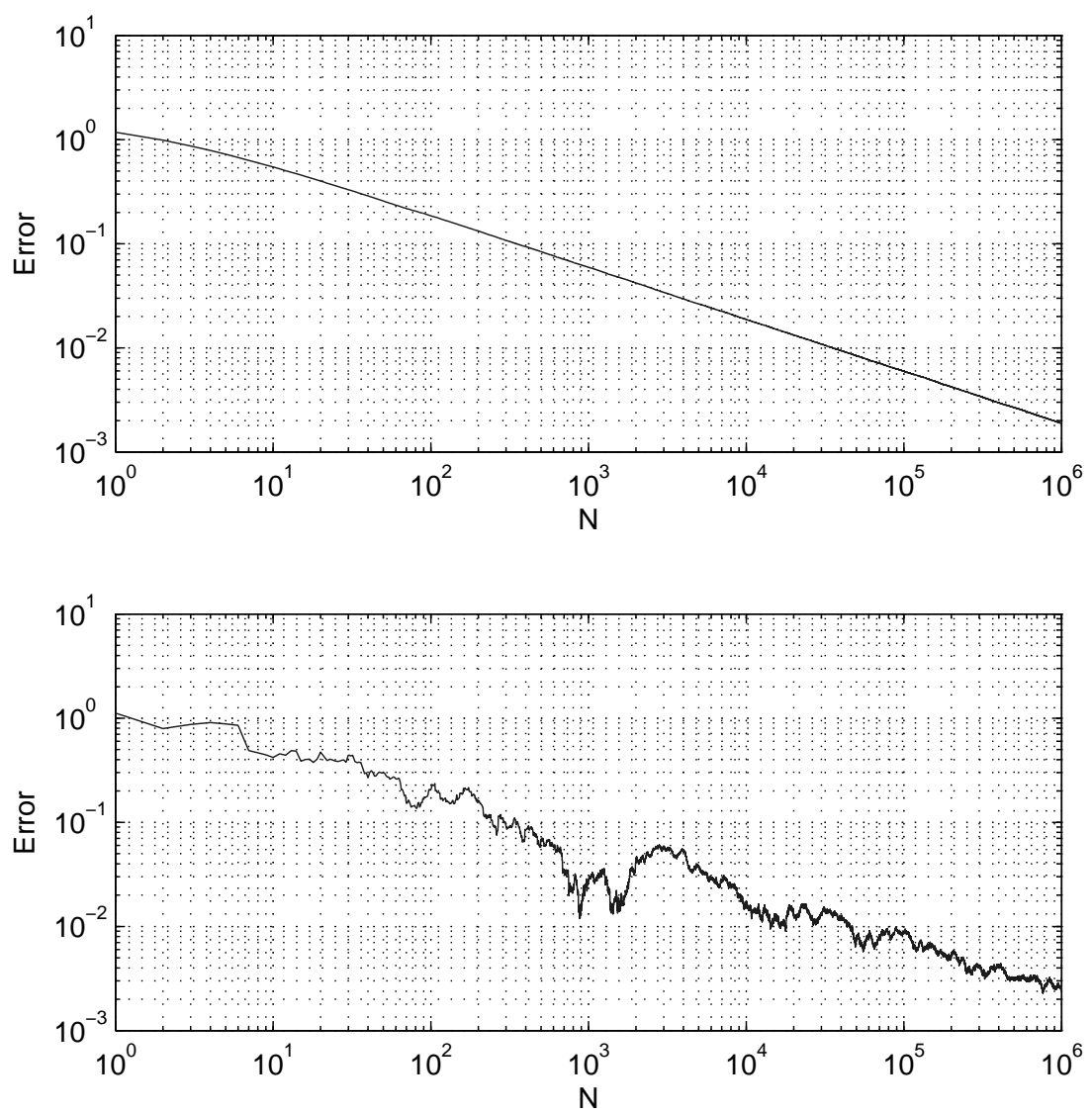

Figure 3.2: Convergence of direct linear Hebbian regression in a 5D space on isotropic white Gaussian input data. Angle between estimated and true parameter vectors (radians) versus number of presented training samples. Top: average over 3000 trials. Bottom: one trial. 


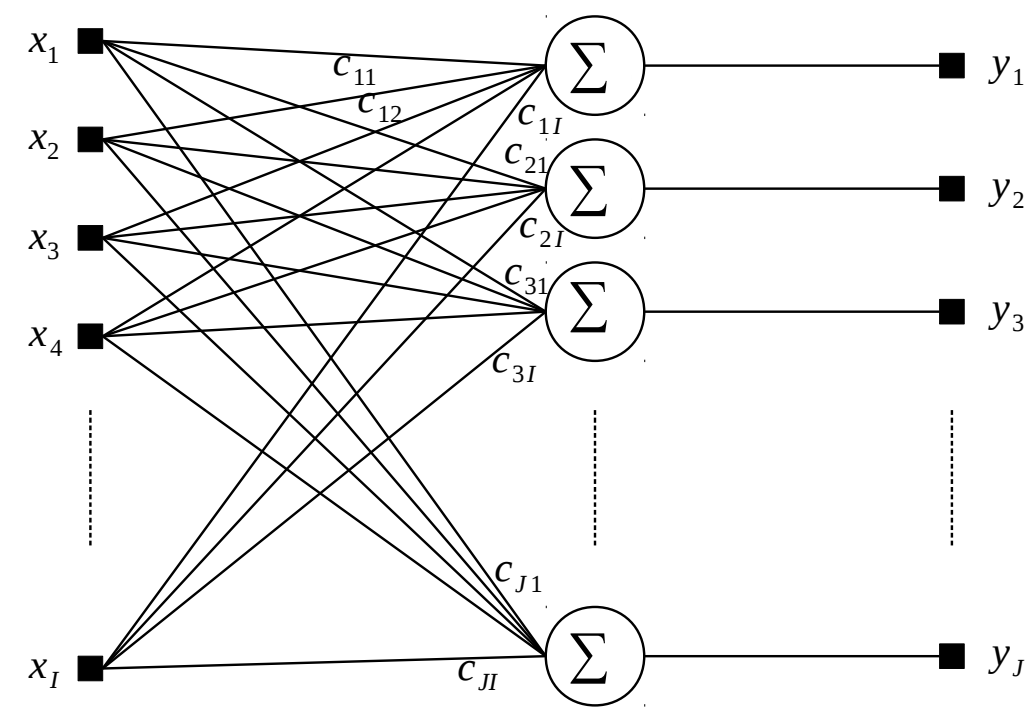

Figure 3.3: Artificial neural network view of associative learning with $I$ inputs and $J$ outputs. The inputs $x_{1}, x_{2}, \cdots, x_{I}$ represent activations of visual features and the outputs $y_{1}, y_{2}, \cdots, y_{J}$ represent activations of actions. The synaptic weights are given by the elements of the matrix $\mathbf{C}: c_{11}, \cdots, c_{J 1}, c_{12}, \cdots, c_{J I}$. 


\section{Chapter 4}

\section{Representation}

Separate from the selection of a learning method, but not independent, is the choice of representation. There is a strong connection between learning method and representation of perceptions and actions. The conventional representation is by the set of measured numbers available from the sensor in question, and representing actions directly by the control signals to be sent to the hardware controller. For a vision system, this conventional representation is a grid of intensity readings from an image sensor.

This may be very efficient for interfacing input and output systems, however, it may not be the representation on which a learning system obtains its best performance. At the other end of the scale, there may be a representation making learning the desired perception-action mapping a trivial task, where there is a direct relation between the action representation and the perception representation.

For the reperesentation, the measured or estimated values themselves may not be sufficient, or, with more information, more sophisticated things can be accomplished. For many applications, some sort of estimate of confidence or reliability of the values is of great value. Any book on estimation theory, e.g. [49], points out the importance of affixing to any estimate of an entity, an estimate of the confidence of said estimate. One example of such a value-confidence pair is the local image orientation representation by complex numbers, where the argument represents the orientation and the magnitude represents the confidence.

In biological vision systems, light intensities measured by photosensitive cells in the retina are not directly transferred to the brain. Some information processing is performed directly in the retina [24]. Regarding processing in the early stages of the visual cortex, experiments were carried out by David Hubel and Torsten Wiesel. They recorded signals from single neurons in the visual cortex of cats and macaques among other animals, see e.g. [22]. Cells were found reacting to visual structures such as edges. The response depends on the orientation of the edge relative to the so called preferred orientation of each such cell. Also, the response fades gradually as the edge orientation is rotaded further away from the preferred orientation of the cell.

This smoothly fading response, the tuning curve, can be utilized such that 
given a few cells with different preferred orientations, the true orientation of the edge can be recovered with greater precision than the number of cells, by comparing relative response strenghts of the different cells. Within computational neuroscience, such a representation is known as population coding. One approach for decoding population codes with equally spaced preferred directions was presented by Deneve et al. [12], however, an implementation of their proposed scheme has shown to bias the decoded value towards preferred orientations or orientations between two preferred orientations.

In computer vision, a similar concept is the channel representation [17]. Motivation for this type of representation is not only biological. Technical advantages of a predecessor to the channel representation were explored by Snippe and Koenderink [47]. Further, there is a strong connection to scale spaces [15]. By specifying the shape of the basis function, also known as kernel or tuning curve, unbiased decoding schemes can be derived [16].

In contrast to the population coding, the channel representation also has a probabilistic interpretation where weighted basis functions are used as a nonparametric distribution representation similar to kernel density estimation. This enables a more solid mathematical approach to handling the representation. See section 4.1.2.

For the channel representation, basis functions are usually placed in a regular grid. Another option is to place new local models when and where there is a need to represent new data and there are no previous models sufficiently close. Such ideas are explored in section 4.2 .

\subsection{The Channel Representation}

Channel representations have originally been suggested as an information representation $[17,47]$. Central to the representation is the basis function or kernel, $b(\cdot)$, corresponding to the tuning curve of population codes $[37,51]$. Several basis functions are distributed with overlap in the space of values to be encoded and a specific value will activate some of these channels. The concatenated list of activations for each channel is the encoded channel vector. The basis function is required to be non-negative, smooth and to have compact support, the later is required for computational efficiency. The smoothness of the basis functions enables recovering of an encoded value with higher precision than the channel spacing. Without noise and with basis functions strictly monotonically decreasing within the support, and away from the center of the basis function, a single encoded value can clearly be recovered with arbitrarily high precision. Several different basis functions have been evaluated including $\cos ^{2}$, B-spline and also Gaussian kernels [16], although the Gaussian kernel lacks compact support. An example of $\cos ^{2}$ basis functions is presented in Fig. 4.1. 


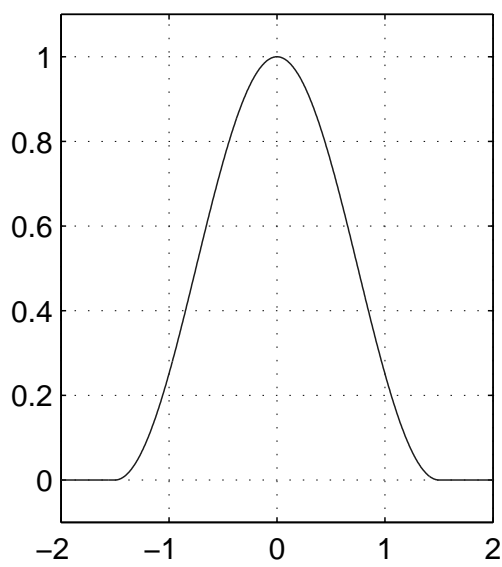

(a)

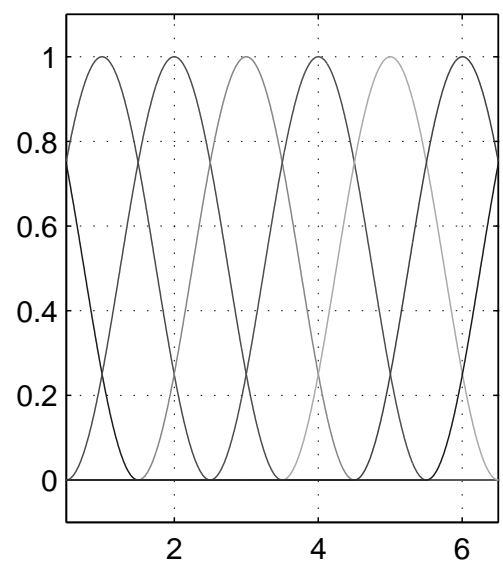

(b)

Figure 4.1: Examples of basis functions for the channel representation with width 3 and three overlapping basis functions. (a) A single basis function. (b) Eight basis functions for representing values in the interval $0.5 \leq \xi \leq 6.5$. The first and the last basis functions have their strongest response outside the interval.

\subsubsection{Channel Encoding}

In order to enable application of discrete signal processing methods, the basis functions are usually placed in a regular grid. For simplicity of presentation, channel encoding of scalar values $\xi$ are first presented. Encoding of vectors then reduces to the matter of combining the separately encoded elements of the vector. Without loss of generality, channel centers are assumed to be at integer positions along the real line.

Assume a basis function $b(\xi)$ fulfilling (for real $\xi$ )

$$
\begin{aligned}
b(\xi) & \geq 0 & & \forall \xi \\
b(\xi) & =0 & & |\xi| \geq w / 2 \\
b(0) & \geq b(\xi) & & \forall \xi \\
b(-\xi) & =b(\xi) & & \forall \xi \\
b(\xi) & >b(\xi+\epsilon) & & 0<\xi<w / 2, \quad \epsilon>0
\end{aligned}
$$

where $w$ is the width of the basis function. The number of simultaneously active channels can be chosen arbitrarily from an encoding point of view, however, three overlapping channels is a de facto standard choice [23], which is motivated by the decoding [16]. From the assumption of integer channel centers follows $w=3$.

With $N$ channels, the encoding of $\xi$ is a vector of channel coefficients

$$
\mathbf{x}=\operatorname{enc}(\xi)=[b(\xi), b(\xi-1), b(\xi-2), \ldots, b(\xi-(N-1))]^{\mathrm{T}} \quad .
$$




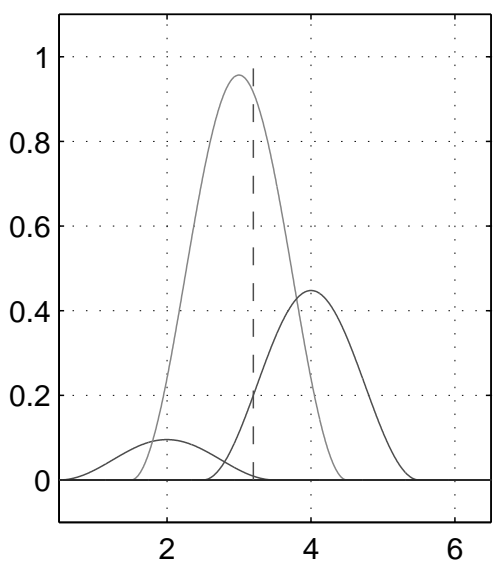

(a)

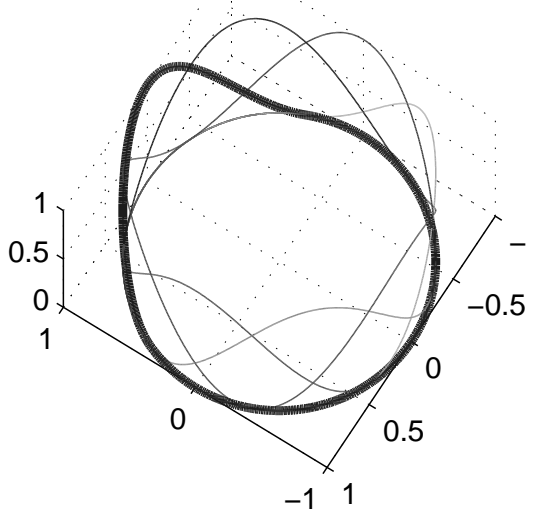

(b)

Figure 4.2: (a) Illustration of a channel vector as weighted basis functions. The encoded value, 3.2, is marked with a dashed line. (b) Illustration of eight basis functions on a modular domain, a circle in this case. One basis function is marked with a bold line.

The valid range, where there are three overlapping channels, is $0.5<\xi<N-1.5$. Within this range, an encoded value can be perfectly recovered in the noise free case. For modular domains such as for representing orientation, the edge channels will wrap around, making the choice of the first channel and corresponding coefficient arbitrary. Fig. 4.2 illustrates a single channel encoded value and channels on a modular domain. In applications, the number of channels, $N$, is first selected depending on the expected noise and characteristics of the values, $\xi$, to be encoded. The domain of $\xi$ is then transformed to the valid encoder range.

For multidimensional data, there are two different approaches [18], both combine the single dimensional channel encodings of each separate dimension of the data. The difference lies in the expected dependencies between dimensions. If the separate dimensions are independent, all dimensions are encoded separately and the resulting vectors are concatenated. If the dimensions are mutually dependent, an outer product of the channel vectors is built, similar to a joint histogram. This corresponds to placing multidimensional channels in an axis aligned grid. This multi-dimensional array of channel coefficients is vectorized and the resulting vector is the channel encoding of a multi-dimensional point. For higher dimensional data, dimensions can be grouped and combined using both metods in any combination that fits the problem at hand. As an example, assume two dimensions to be dependent and the remaining dimensions mutually independent and independet of this two dimensional subspace. A suitable encoding is to combine the two mutually dependent dimensions using the vectorized outer product and concatenating 
the independently encoded channel vectors of the remaining dimensions with the channel vector of the two dimensional space.

The drawback of the joint channel representation is the exponential growth of the number of coefficients with the number of dimensions. This is mitigated by sparse data structures, as the number of non-zero elements are at most three to the power of the dimensionality of $\xi$.

Up to here, only single, but possibly multi-dimensional, values have been encoded. However, the full benefits of the channel representation only appear when combining multiple encoded values of the same entity. The combination operation is usually the mean vector of several encoded measurements $\xi_{1}, \ldots, \xi_{I}$ :

$$
\overline{\mathbf{x}}=\frac{1}{I} \sum_{i=1}^{I} \mathbf{x}_{i}=\frac{1}{I} \sum_{i=1}^{I} \operatorname{enc}\left(\xi_{i}\right)
$$

Depending on the view of the channel representation, the entity $\overline{\mathbf{x}}$ will be interpreted slightly differently.

\subsubsection{Views of the Channel Vector}

Over the years, different views of the channel representation have evolved. Initially, the channel representation was suggested as representation, primarily for sensor data. In [17], Granlund presents an example with spatially limited channels distributed in the image plane, where each channel also has a preferred orientation. As mentioned in the introduction, the inspiration originates from biological vision systems. An idea similar to this example is a bank of Gabor filters.

A slightly different view of the channel vector, especially the mean channel vector (4.7), is that of a soft histogram. For a soft histogram, each sample is distributed in several bins, the distribution of the sample depends on a kernel or basis function. With this view, each channel coefficient corresponds to a bin. The advantage is a possibility to determine properties, such as the mean of a mode, with higer precision than the channel spacing. A regular histogram is obtained if a box basis function is used, which is one between -0.5 and 0.5 and zero everywhere else. The advantages of a soft histogram is similar to those of the Averaged Shifted Histogram by Scott [45].

The aim of Scott was to generate better distribution estimations than those obtained from histograms without the computational demands of kernel density estimators. This leads to the final view of the channel vector, that of the channel representation as a method for distribution estimation and for non-parametric distribution representation. It can be shown that the expected value of each channel coefficient is the probability density function for $\xi$ convolved with the basis function, and evaluated at each channel center. Assuming a symmetric and properly scaled basis function, the mean channel vector (4.7) is the sampled kernel density estimate of the distribution of $\xi$. 


\subsubsection{The $\cos ^{2}$ Basis Function}

As previosly mentioned, different basis functions have been proposed and evaluated. For the biologically inspired information representation, the exact shape of the basis function or tuning curve does not notably affect the performance of the system [12]. For the probabilistic view and for decoding, the shape of the basis function has a larger impact. The proper decoding scheme also depends on the choice of basis function, thus the specific basis function used will be presented before the decoding of the channel representation.

In this work, $\cos ^{2}$ basis functions are used:

$$
b(\xi)=\left\{\begin{array}{ll}
\cos ^{2}\left(\frac{\pi \xi}{w}\right) & |\xi|<\frac{w}{2} \\
0 & |\xi| \geq \frac{w}{2}
\end{array} .\right.
$$

The parameter $w$ sets the width of the support of the basis function. For integer channel centers and three overlapping channels $w=3$, see figure 4.1.

It can be shown that the $\cos ^{2}$ channel representation possesses certain properties concerning the $L_{1}$ and $L_{2}$ norms [16]. Specifically, these norms are constant for channel vector of a single encoded value within the valid range of the encoding,

$$
\|\operatorname{enc}(\xi)\|_{1}=\frac{3}{2}
$$

for $0.5<\xi<N-1.5$. The total activation is constant independent of the value of $\xi$ with respect to the choice of channel centers. This does not hold for Gaussian channels, where the $L_{1}$ norm varies with the relative position of $\xi$ with respect to channel centers. For $\cos ^{2}$ channels also

$$
\|\operatorname{enc}(\xi)\|_{2}=\frac{3}{2 \sqrt{2}}
$$

hold for $0.5<\xi<N-1.5$. This sets the $\cos ^{2}$ channels apart from the B-spline channels. For a sum of channel vectors, the $L_{1}$ norm is clearly proportional to the number of samples while the $L_{2}$ norm can be shown to depend both on the number of samples as well as the spread of the samples.

\subsubsection{Robust Decoding}

Decoding of channel vectors is dependent on the selection of basis function. Forssén has presented a decoding scheme for $\cos ^{2}$ channels [16] which will be presented here. The decoding scheme is unbiased when the encoded values belong to the same decoding window. Possibilities for unbiased decoding when this is not the case are to be further investigated.

Since one of the fundamental ideas behind the channel representation is the possibility of representing multimodal entities, non-zero elements of the channel vector not belonging to the mode to be decoded should not affect the decoding. Thus, only a part of the channel vector should be considered, the decoding window.

Let one element $x_{l+0}$ be the first element in the decoding window of a channel vector where a value $\xi$ has been encoded. Let $r>0$ be a parameter representing 
a uniform scaling of the channel vector, such that

$$
x_{l+q}=r b(\xi-l-q)=r \cos ^{2}\left(\frac{\pi}{w}(\xi-l-q)\right)
$$

where it is assumed that $\xi$ is within the active region of each channel within the decoding window.

The equation can be reformulated as [16]

$$
x_{l+q}=\frac{1}{2}\left(\begin{array}{lll}
\cos \left(\frac{2 \pi}{w} q\right) & \sin \left(\frac{2 \pi}{w} q\right) & 1
\end{array}\right)\left(\begin{array}{c}
r \cos \left(\frac{2 \pi}{w}(\xi-l)\right) \\
r \sin \left(\frac{2 \pi}{w}(\xi-l)\right) \\
r
\end{array}\right)
$$

and $Q$ of these equations can be stacked, forming a linear equation system

$$
\left(\begin{array}{c}
x_{l} \\
x_{l+1} \\
\vdots \\
x_{l+Q-1}
\end{array}\right)=\frac{1}{2}\left(\begin{array}{ccc}
\cos \left(\frac{2 \pi}{w} 0\right) & \sin \left(\frac{2 \pi}{w} 0\right) & 1 \\
\cos \left(\frac{2 \pi}{w} 1\right) & \sin \left(\frac{2 \pi}{w} 1\right) & 1 \\
\vdots & \vdots & \vdots \\
\cos \left(\frac{2 \pi}{w}(Q-1)\right) & \sin \left(\frac{2 \pi}{w}(Q-1)\right) & 1
\end{array}\right)\left(\begin{array}{c}
r \cos \left(\frac{2 \pi}{w}(\xi-l)\right) \\
r \sin \left(\frac{2 \pi}{w}(\xi-l)\right) \\
r
\end{array}\right)
$$

From this it is clear that choosing a decoding window of size $Q=3$ will generate an equation system neither under- nor over-determined. Further, it is clear that choosing three overlapping channels, $w=3$, will make sure that it is possible to choose the decoding window such that the assumption regarding all channels being active is fulfilled. Choosing $w<3$ may break the assumption depending on the position of the mode relative to channels centers. From $w=3$ it also follows that no other channel outside the decoding window is activated by the decoded $\xi$. Similarly, this is not certain for $w>3$.

Inserting these parameters reduces (4.13) to

$$
\left(\begin{array}{c}
x_{l} \\
x_{l+1} \\
x_{l+2}
\end{array}\right)=\frac{1}{2}\left(\begin{array}{ccc}
1 & 0 & 1 \\
-\frac{1}{2} & \frac{\sqrt{3}}{2} & 1 \\
-\frac{1}{2} & -\frac{\sqrt{3}}{2} & 1
\end{array}\right)\left(\begin{array}{c}
r \cos \left(\frac{2 \pi}{3}(\xi-l)\right) \\
r \sin \left(\frac{2 \pi}{3}(\xi-l)\right) \\
r
\end{array}\right)
$$

with the solution

$$
\left(\begin{array}{cc}
r_{1} & \cos \left(\frac{2 \pi}{3}(\xi-l)\right) \\
r_{1} \sin \left(\frac{2 \pi}{3}(\xi-l)\right) \\
r_{2}
\end{array}\right)=\frac{2}{3}\left(\begin{array}{ccc}
2 & -1 & -1 \\
0 & \sqrt{3} & -\sqrt{3} \\
1 & 1 & 1
\end{array}\right)\left(\begin{array}{c}
x_{l} \\
x_{l+1} \\
x_{l+2}
\end{array}\right)
$$

from which $\xi, r_{1}$ and $r_{2}$ can be obtained. Note that for one single encoded value $r=r_{1}=r_{2}$ but for multiple encoded values, $r_{1}<r_{2}$ in general. The value of $r_{2}$ is directly proportional to the number of samples within the decoding window and is referred to as evidence in paper $\mathrm{C}$. The relation between $r_{1}$ and $r_{2}$ depends on the distribution of encoded points within the decoding window and is further discussed in said paper. Finally, a geometric interpretation of these entities is possible, however that is the subject of future work.

Usually, the decoding window corresponding to the largeset evidence is selected. This selection procedure reduces to filtering with a three element box filter and picking the index of the largest response. If required by the application, other modes of a multimodal channel vector can be decoded. 


\subsection{Mixtures of Local Models}

An approach in some ways similar to the channel representation is to place simple and different local models in the space to be represented. The difference lies in the placement of the basis functions and local models. Channel basis functions are usually placed beforehand, while local models are usually placed when and where it is required by the traing data.

The common issue is the selection of the size of the basis functions or the local models. Additionally, for local models, issues such as when and where to place new models and how old models should be updated need to be addressed. For online systems with local models, the growth of the number of local models need to be regulated to avoid depletion of memory and to limit the time needed for prediction.

\subsubsection{Tree Based Sectioning}

A selection of different approaches has been proposed. One approach is the tree based sectioning of the input space employed by decision and regression trees, described in section 3.4. Each node in the tree splits the current part of the input space into two subparts. The local models live in the leaves, corresponding to distinct regions in the input space.

The tree structure makes finding the correct local model a quick operation, proportional to the depth of the tree and logarithmic to the number of local models for a balanced tree. However, the tree structure also makes finding the neigbours of a particular model cumbersome. Thus, for regression trees there is typically no smooth interpolation between local model centra.

\subsubsection{Weighted Local Models}

Another approach is to interpolate the predictions of a set of local models. Three methods will be used to illustrate this approach. Two are named locally weighted regression but abbreviated loass [7] and LWR [43] respectively. The third method is the successor of LWR: locally weighted projection regression, LWPR [50].

LWPR differs from loass and LWR in when the local models are created. LWR and loass simply store all training data during learning, so called lazy learning. A local model is created when requesting a prediction for a new point. Loass uses the $q$ nearest neighbours to generate a model, weighted by a weight function scaled depending on the distance to the $q$ th closest point. LWR uses all points for model estimation and a Gaussian weight function is proposed, where the width of the Gaussian is a global parameter. For both methods, a new model is estimated for every query.

On the other hand, LWPR generates models during training. This approach has two main advantages: all training data need not to be stored and queries are possibly answered faster as no new models need to be estimated. The question is when to generate a new model and when to use a training data sample to update an old model. In LWPR a new model is created when the weight of all other 
models, evaluated at the new sample, is under a set threshold. See section 3.3.

All these three methods can be seen as interpolation of local models. In the case of loass and LWR, the local models are simple (each training sample is a model) but the interpolation is complex, interpolation involves weighting samples depending on the query point and estimating a new local model. In LWPR, the most demanding part is shifted from prediction to learning. Interpolation is a weighted mean of the closest models, however, each local model is more complex.

The interpolation of local models also leads to a partitioning of the input space in a sense, however, algorithmically these methods differ significantly from the tree based regression methods. The interpolation of local models comes with the cost of evaluating the distance from the query point to all local models in LWPR. The time complexity thus increases linearly with the number of models. 


\section{Chapter 5}

\section{Experiment Highlights}

Methods from chapter 3 have been used to solve problems presented in chapter 2 . In this chapter, some outcomes of these experiments are summarized. Full results are available in the included publications. The opportunity is however taken to present comments regarding relations between the different experiments which are not available in the publications. Three main problems are addressed: vision based autonomous driving, inverse kinematics and dynamic system control.

One of the primary differences between these problems is the source and quality of training data. For vision based autonomous driving, self-evaluation is not possible by the system and thus training data have to be generated externally. For the dynamic system controller, the success of an action is available to the learning system so self-learning is possible. However, with high quality externally generated training data, the learning rate can be increased. Finally, for the inverse kinematics problem, the internally generated training data from the directed exploration are sufficient for fast learning. External training data may however be provided to the system as to select preferred solutions for a redundant robotic manipulator.

\subsection{Vision Based Autonomous Driving}

The ability of the systems to learn vision based autonomous driving is primarily evaluated on a reconfigurable indoor track, figure 5.4(e). Three main questions are explored: if the system can learn to drive around the track at all, how robust the system is to track changes and how fast training data is processed. Reconfiguration of the track also provides an indication of whether the system mainly picks up features on the track or if it picks up features from the surrondings. Table 5.1 summarizes the evaluated approaches for autonomous driving.

Three different variants are evaluated in this work: offline learning using random forest regression, online learning using LWPR and online learning using qHebb, a channel based associative learning algorithm. The possibilities of using convolutional networks were evaluated by Schmiterlöw [44]. 


\begin{tabular}{|l|l|l|}
\hline Method & Successful Driving & Training Data Proc. Speed \\
\hline Conv. Networks [44] & No, (offline predictions) & Days (batch) \\
Random Forest [13] & Yes & Hours (batch) \\
LWPR [33] & With input projection & Init. video rate, slows down \\
Assoc. Hebbian [31] & No & Video rate (online) \\
Weighted qHebb [31] & Yes, dynamic track & Video rate (online) \\
\hline
\end{tabular}

Table 5.1: Summary of approaches for vision based autonomous driving.

\subsubsection{Random Forest Regression}

Using random forest regression, it is possible to learn a mapping from low level image features to steering signal that will keep the vehicle on the road [13]. The system shows generalization abilities such as driving on one track configuration when trained on another, given that the training track contains all track elements as required on the evaluation track. A system trained on a track with only left turns was not able to navigate a track with turns to the right.

The random forest learning system does not use all image features for steering signal prediction. Only a few fetures are used for decisions in the visited inner nodes of each tree. By recording the spatial positions of the features involved in decisions, heat maps can be generated indicating where in the image the essential information for steering signal prediction is located. Four frames of this type are shown in figure 5.1. Clearly, the system bases its decisions mainly on the road markings.

On the other hand, there were training sets from which the system was not able to learn to navigate not even the same track layout. Particularly, each successful training set has to contain examples of driving back onto the correct path from a selection of erroneous poses slightly off the desired path. Also, the training data set should not contain examples where the vehicle is driven from the desired path to such erroneous poses. Collection of training data thus had to be momentarily stopped while positioning the vehicle in an erroneous pose.

\subsubsection{Locally Weighted Projection Regression}

With an online approach, or, more precisely: with an approach which does not slow down below video rate before enough training data have been collected, generation of training data is simplified. The effect of training on a particular training set can be evaluated immediately. Any additional examples of driving back onto the correct path can be seamlessly collected on demand.

As noted in section 3.3, LWPR is not video rate capable using the full 2048 dimensional visual feature vector. For these experiments, input vectors are projected onto a 512 dimensional subspace. The projection operation was found by a principal component analysis of feature vectors recorded during manual driving around the indoor track, clockwise as well as counter clockwise. Additionally, care 

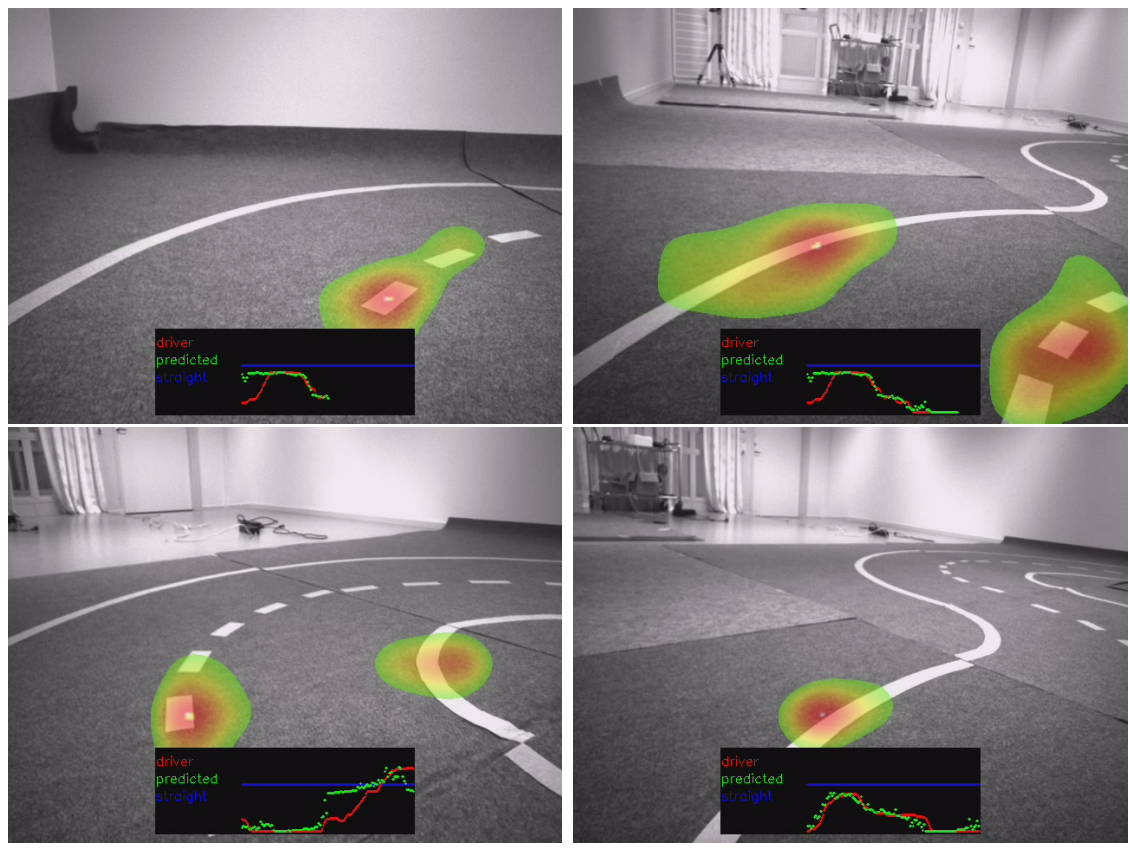

Figure 5.1: Illustration of the Random Forest activation when navigating around the P-shaped track. The marked regions denotes visual areas that were mostly used by the regressor for predicting steering. (Paper A, Fig. 2) 


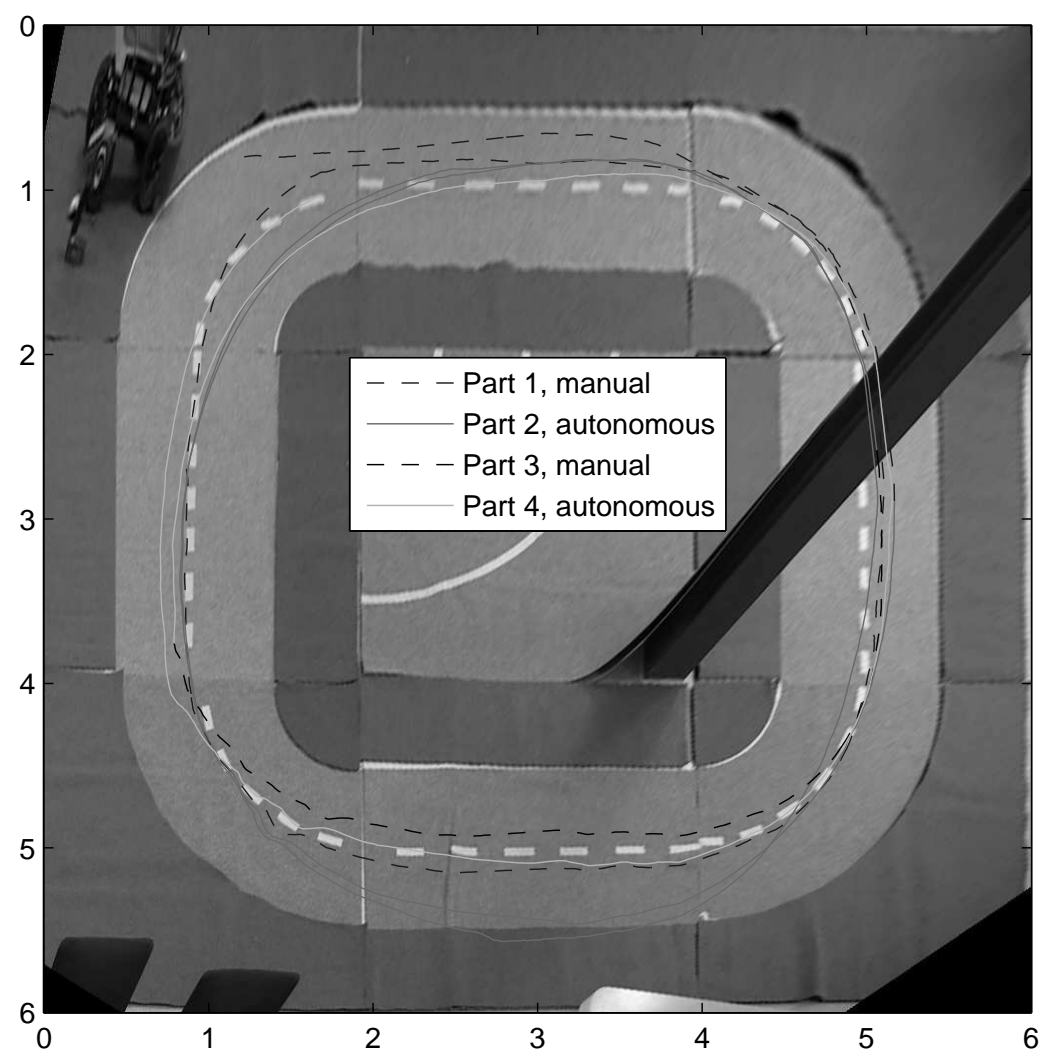

Figure 5.2: Trace of first five clock-wise laps for a previously untrained system overlaid on an image of the track reprojected onto the ground coordinate system, with coordinates in meters.

had to be taken to limit the number of local models.

The best illustration of the performance of the system is provided by the supplementary video ${ }^{1}$. A selection of ground projected frames with overlaid vehicle paths from the video is provided here. In figure 5.2, the system is manually driven through five corners whereafter manual control is released. The system then successfully drives around the track, however, there is a tendency to drive slightly off the road on the bottom straight. By manually controlling the system for half a lap, the unwanted behavior is corrected. The system, now trained on right turns, is turned around and manually driven through three of the four left turns. The forth, unseen corner, is successfully navigated autonomously, see figure 5.3. At the end of the sequence, the road markings are modified while the system continues

\footnotetext{
${ }^{1}$ Available at http://users.isy.liu.se/cvl/ofjall/onlinePerceptionActionLearningSmaller.mp4
} 


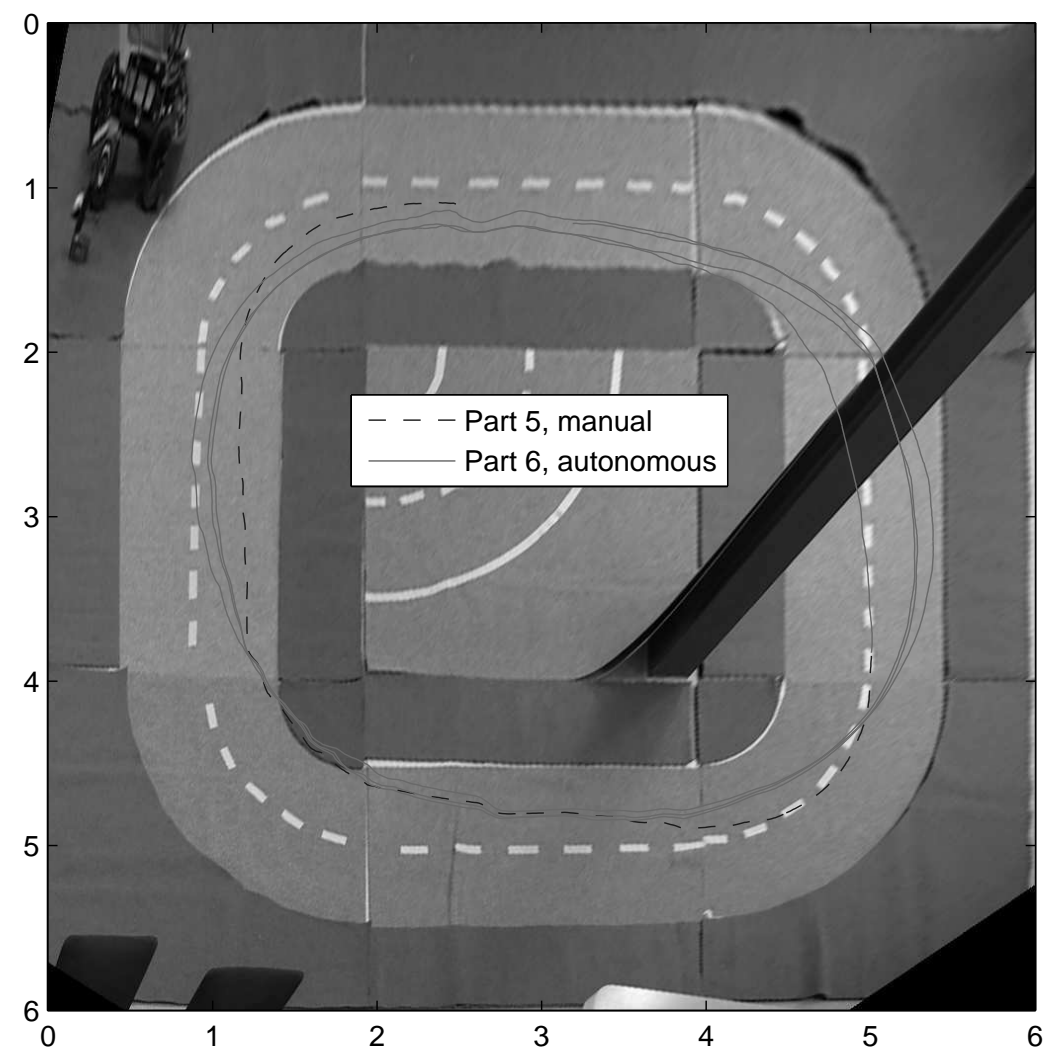

Figure 5.3: Trace of the four laps following Fig. 5.2 where the robot runs counter clockwise. 


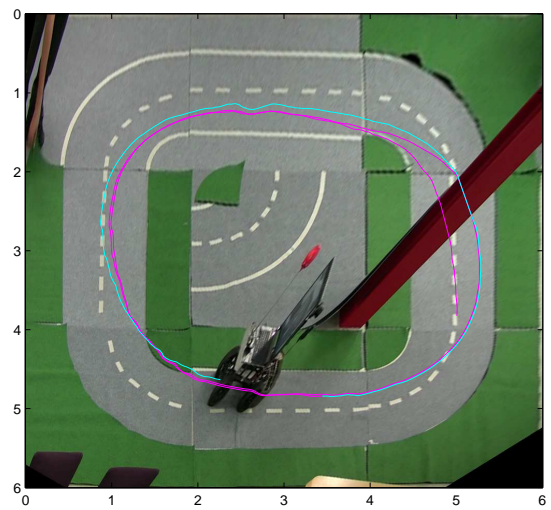

(a) Part 7

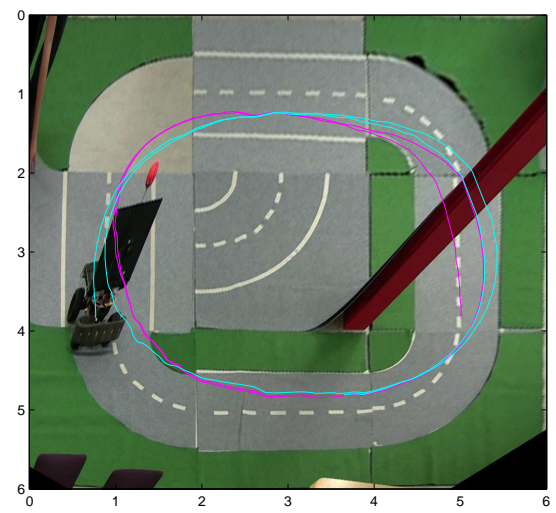

(c) Part 9

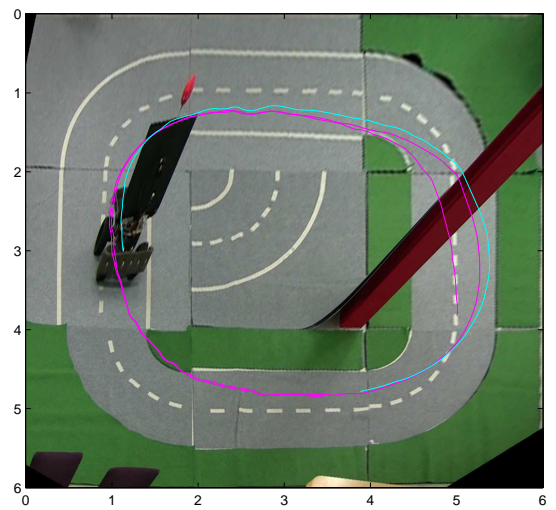

(b) Part 8

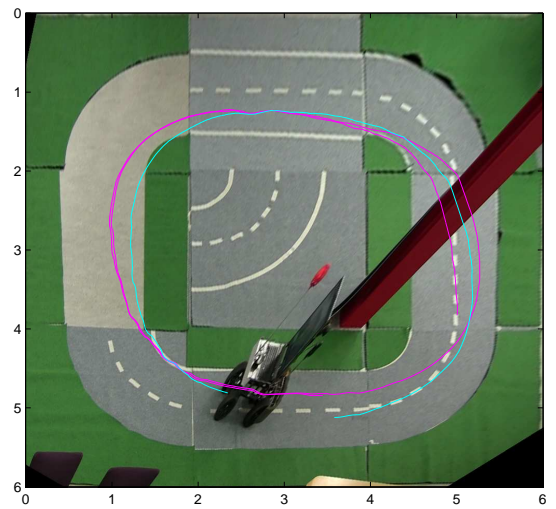

(d) Part 10

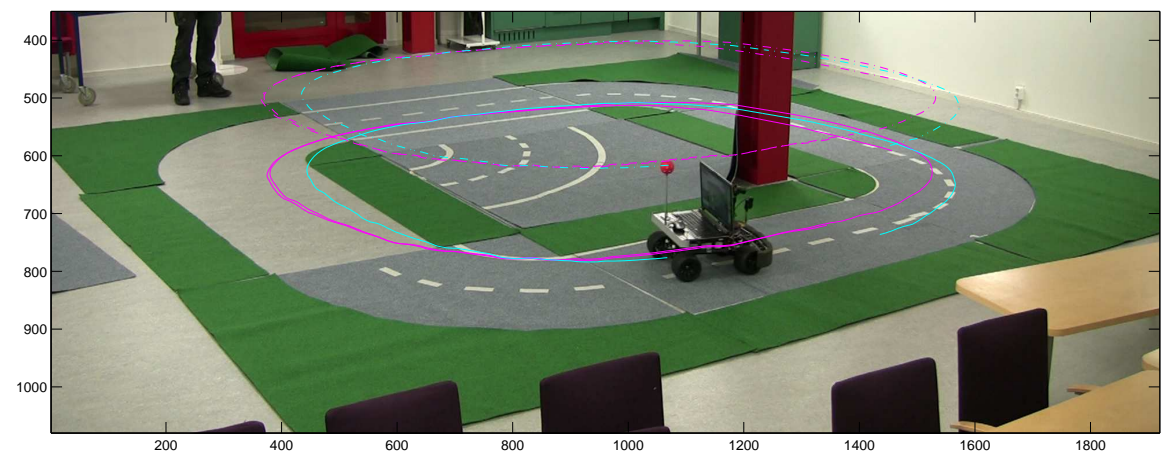

(e) Part 10, original view with red marker trajectory (dashed) and the corresponding ground projection (solid).

Figure 5.4: Autonomous trajectories by the robot while reconfiguring the track (cyan), with last laps on unmodified track for comparison (magenta). 


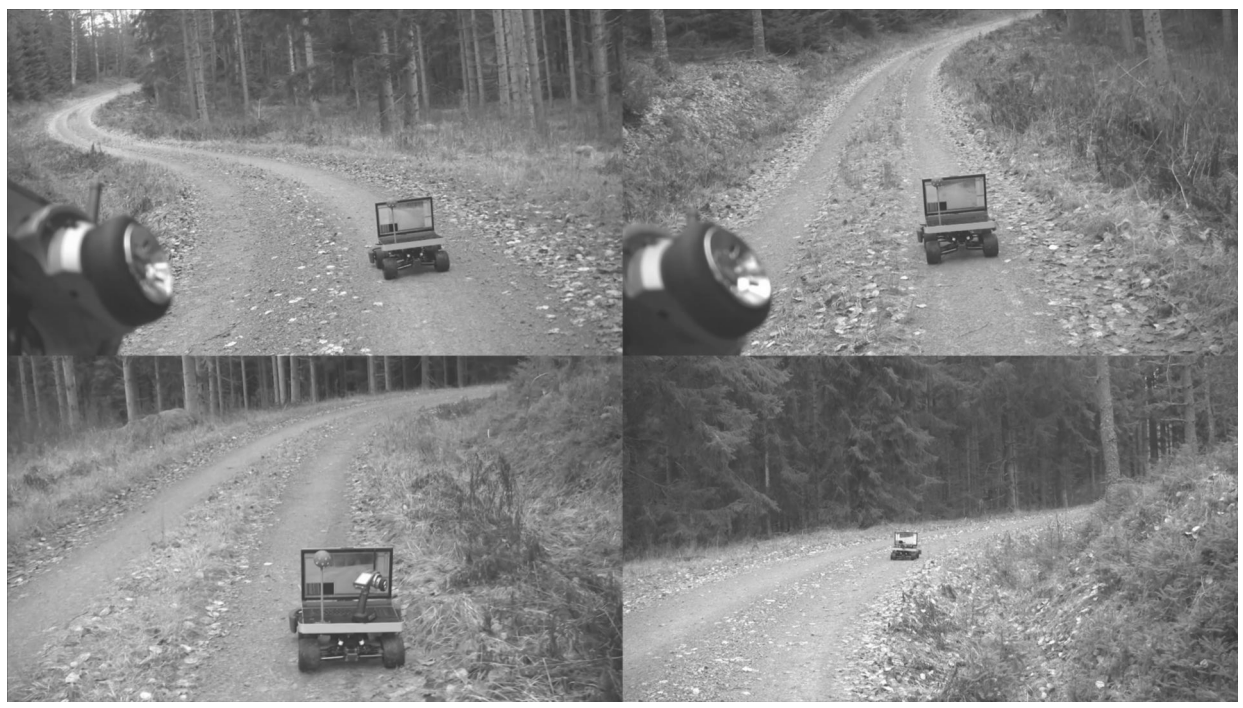

Figure 5.5: Robot driving autonomously on a forest road. The video is available at http://users.isy.liu.se/cvl/ofjall/onlinePerceptionActionLearningSmaller.mp 4

to successfully follow the track, figure 5.4.

An additional experiment was performed where the system was trained outdoors on a forest road. After two minutes of manual control, the system was able to follow the road autonomously. Four frames from the video are shown in figure 5.5. Note that no changes were made to the system between the indoor and outdoor experiments. The PCA input feature dimensionality reduction computed from an indoor sequence proved general enough for outdoor driving.

\subsubsection{Extended Hebbian Associative Learning: qHebb}

The Hebbian associative learning approach presented in section 3.5 is extended with a novel update scheme decoupling learning and forgetting rates, and an input weighting scheme which emphasizes input features expected to provide more specific information regarding the output. These extensions enable learning of autonomous driving by channel based Hebbian associative learning.

The supplementary video demonstrates the system ${ }^{2}$. Frames from the video are shown in figure 5.6. Compared to the LWPR approach, qHebb requires less training time. The vehicle is manually controlled through two corners followed by short corrections during the following two corners. After this, the vehicle autonomously follows the track, even during track reconfiguration.

The primary advantage of the channel based associative learning is the ability to handle multimodal outputs, where a specific input configuration can be associated with two or more different output values without introducing averaging

\footnotetext{
${ }^{2}$ Available at http://users.isy.liu.se/cvl/ofjall/bmvc2014.mp 4
} 
between the output modes. This is illustrated in the second part of the supplementary video. A multimodal output is introduced when the vehicle is manually turned right at an intersection where it was driven straight during initial training. The two modes are visible during the following laps when the vehicle either goes straight or turns right at the intersection, depending on which mode gets a slightly stronger response.

\section{$5.2 \quad$ Inverse Kinematics}

The inverse kinematics problem is solved using a self-learning, explorative approach. Locally weighted projection regression is used for learning and LevenbergMarquardt [26] minimization of the pose error is used for targeted exploration.

Two variations of the system are evaluated. The first approach is a naive approach where the best prediction from the learning system is used as an initial iteration point for pose error minimization and where each iteration is used as training data. Each algorithm is used alone and all communication is through the conventional inputs and outputs of each method. Particularly, the robot has to be moved for estimating derivatives of the pose error surface.

In the second approach, the integrated approach, the subsystems are more closely connected. Estimates of derivatives, as required by the pose minimization, are obtained from the partial inverse kinematics model learned by the learning system.

\subsubsection{Seven DoF Robotic Manipulator}

The system was implemented on a KUKA LWR robotic arm in an application for active inspection. The system was able to learn an inverse kinematics model of the relevant area from exploration. Previous training data consisted of one point, the configuration and pose of the robotic arm at the start of the experiment.

A selection of frames from the supplementary video ${ }^{3}$ are presented in figure 5.7. The selected sequence shows exploration for reaching an overview pose when only close range poses are previously known by the system. In the video, the learning approach is compared to the built-in inverse kinematics of the robotic arm. Since the built-in approach uses a pre-specified model of the robot, solutions can be calculated directly. However, this requires an accurate geometrical model of the robot and the robot will stop if it approaches a singular point, where the rotation axes of two joints coincide.

Since only one pose measuring system is available, the reported pose from the robot controller, an evaluation of the precision of the full system cannot be performed. The pose minimization can be continued until the controller reports an arbitrarily small pose error. For the current experiment, minimization was stopped when the position error was below $0.1 \mathrm{~mm}$ and the angular error was below 0.001 radians, corresponding to $0.1 \mathrm{~mm}$ at $100 \mathrm{~mm}$ from the rotation axis.

\footnotetext{
${ }^{3}$ Available at http://users.isy.liu.se/cvl/ofjall/onlinePerceptionActionLearningSmaller.mp 4
} 


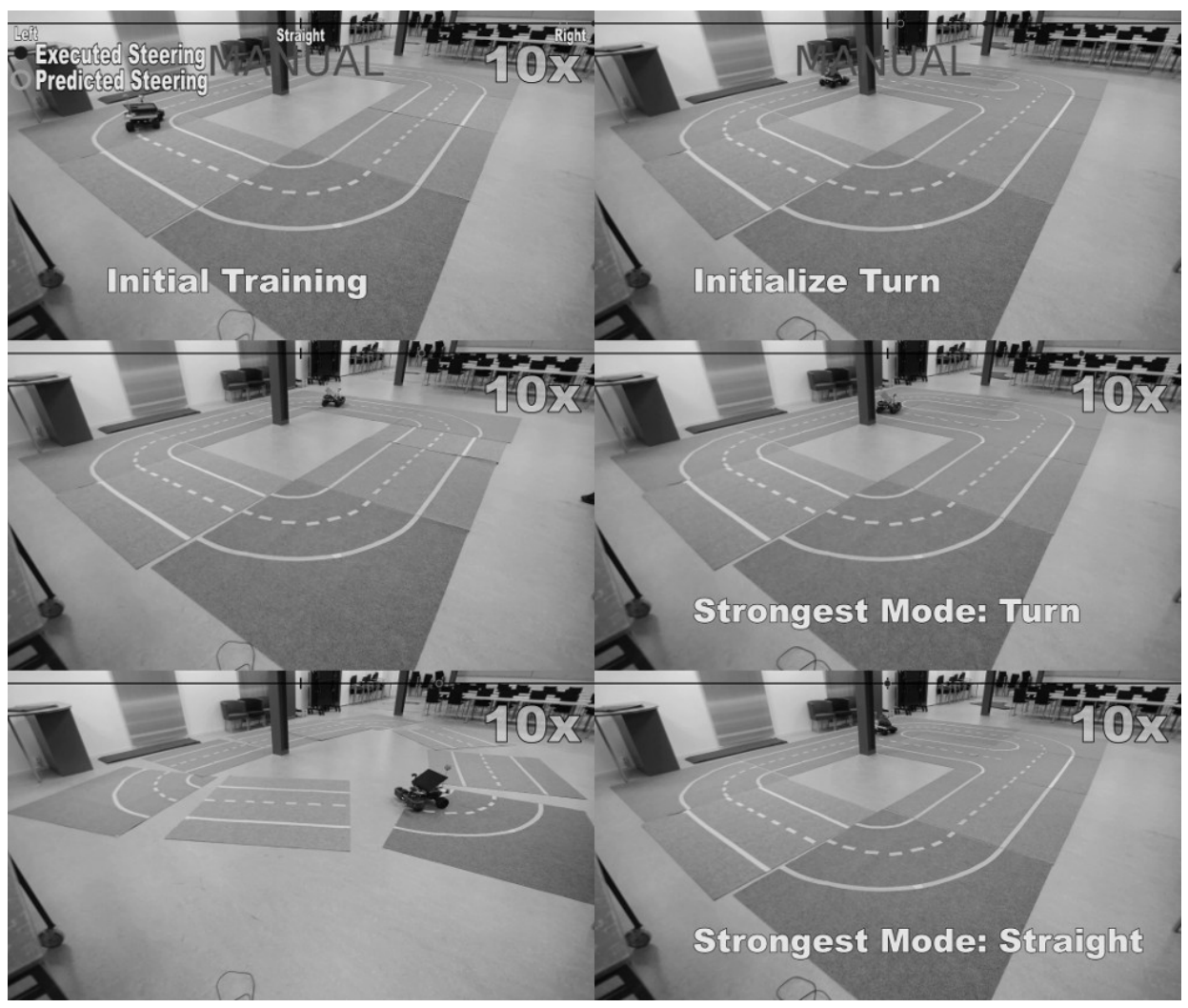

Figure 5.6: An autonomous vehicle learns a mapping from general low level image features to steering signal from demonstration by remotely operating the vehicle during parts of the first lap. When the remote override is released, the vehicle seamlessly continues autonomously. After the training period, the system showed robustness towards reconfiguration of the track (left column). After introducing an intersection, the vehicle can be forced to take the other way by a short application of manual control. After this new training data is acquired, both modes, going straight and turning, are present in the system. When the vehicle reaches the intersection again, it either goes straight or turns depending on which mode happen to get a stronger response (right column). However, the modes are never mixed, which would be noticed by the vehicle making half a turn at the intersection. The figure shows a selection of frames from the supplementary video available at http://users.isy.liu.se/cvl/ofjall/bmvc2014.mp4. 


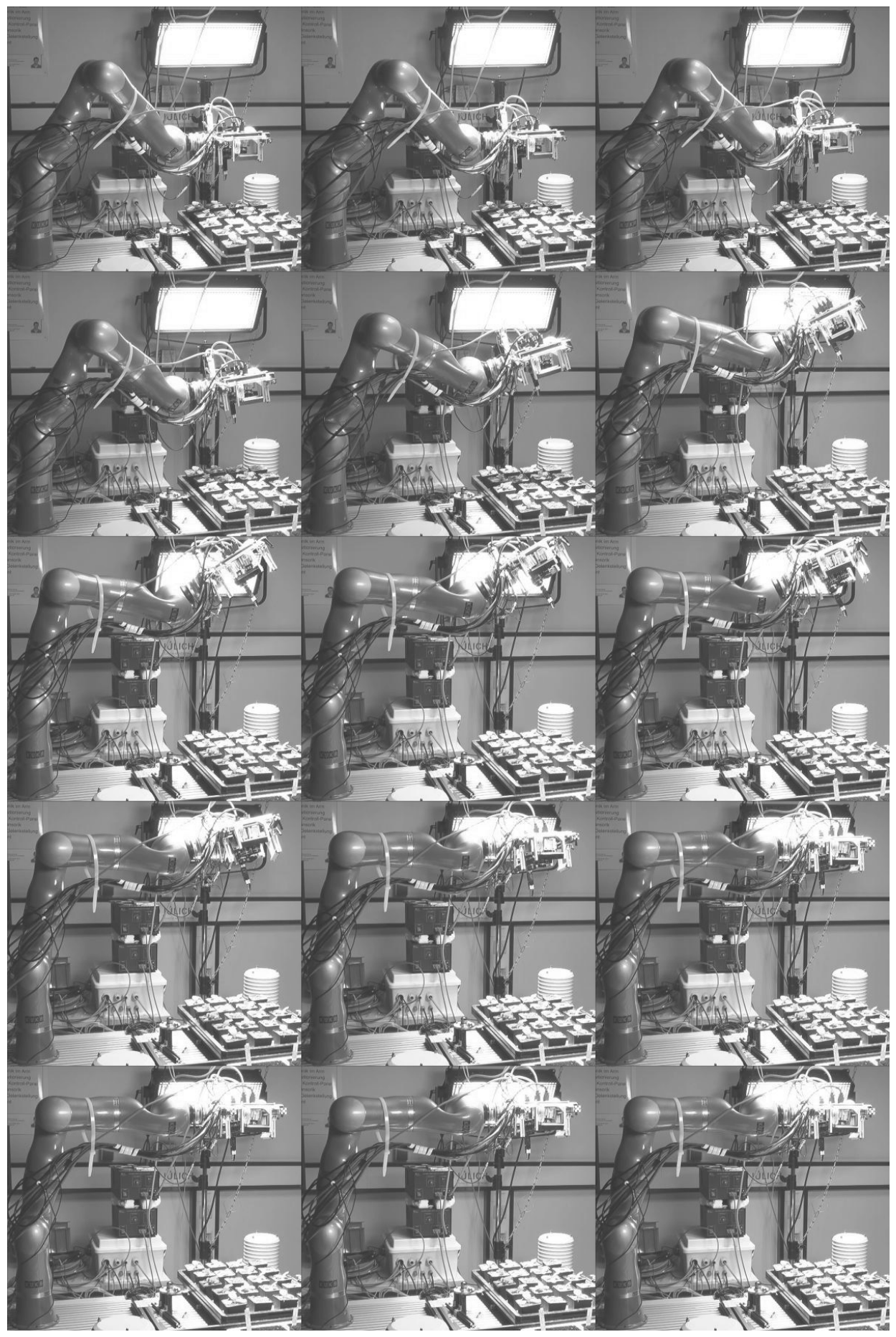

Figure 5.7: Temporally equidistant frames from a video demonstrating exploration for learning inverse kinematics. The full video is available at http://users.isy.liu.se/cvl/ofjall/onlinePerceptionActionLearningSmaller.mp 4 


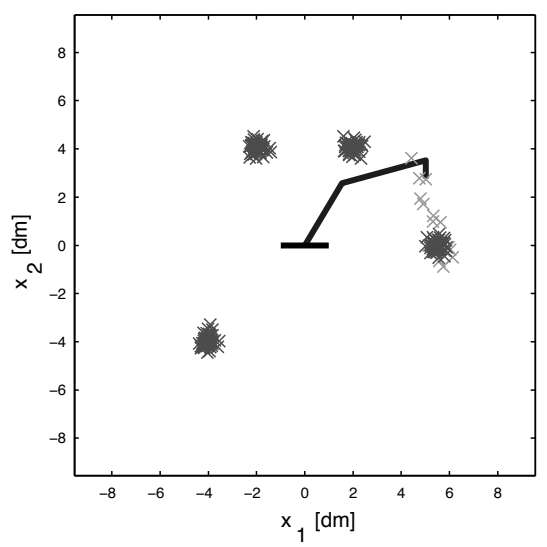

Figure 5.8: The simulated robotic arm and the clusters of evaluation points.

\subsubsection{Synthetic Evaluation}

An experiment on a simulated robotic arm has been performed in order to obtain comparable results. The simulated planar arm has three joints and the desired pose is the two dimensional position of the tip of the last link. The evaluated method controls the arm by specifying the three joint angles and the simulation return the position of the final link with added Gaussian noise.

The evaluation set consists of 200 points in four clusters where point $n$ is in cluster $(n \bmod 4)$. Figure 5.8 illustrates the clusters and the robotic arm. During evaluation, the robotic arm is to reach each pose, one pose at a time, in order, thus changing cluster for each new pose. When the first evaluation point in a cluster far from the known areas is to be reached, the system is expected to require some iterations to move from the closest previously visited area to the desired position. When the arm is supposed to return to the second point within the same cluster, the number of iterations required is expected to be lower. After visiting a few points within the cluster, the system is expected to be able to move directly to the desired pose.

The evaluation results for the two variants are shown in figure 5.9. The graphs show the number of iterations required to reach each test point for the naive combination and the integrated method respectively. Independent zero mean Gaussian noise with standard deviation 0.03 is added to the position estimates. This corresponds to $0.2 \%$ of the diameter of the space reachable by the robot. The shown results are the average of ten runs and in general, the number of iterations required to reach a particular point decays with increasing number of visited points in the same cluster. The density of points at the end of the evaluations indicate that the integrated method requires fewer movements of the robot to reach a particular 

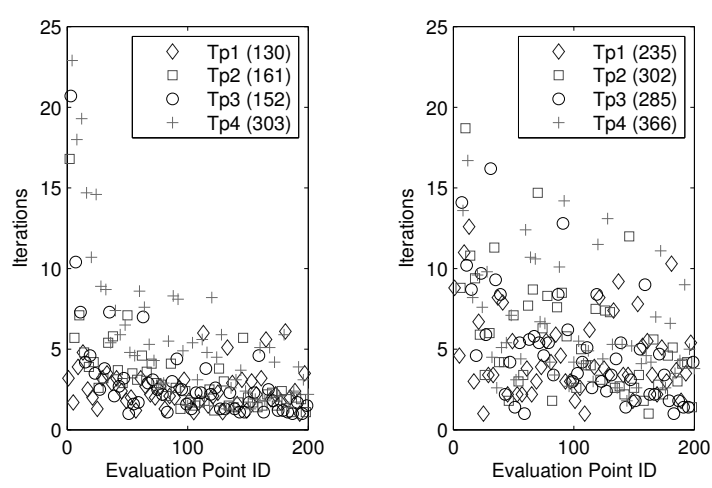

Figure 5.9: Mean number of robot arm movements required to reach each evaluation point. (Left) Integrated method; (Right) Naive combination.

pose.

Figures 5.10 and 5.11 illustrate sequences of reaching for four different points, (a), (b), (c) and (d), in that order. Each system is initially provided with a training data set covering a small area of the reachable space. Visited points are marked by crosses and the valid area of each local model is marked by a circle. A small circle indicate the initial pose provided by the learning system before pose error minimization. As can be seen, new local models are created as the arm reaches areas not covered by previous models. In figure 5.11, derivatives are numerically estimated by small motions of the arm. In figure 5.10, derivatives for the pose error minimization are obtained from the partially learnt model. As visible in figure 5.10, visited points are more widely spread, especially around target poses outside the previously known area. This indicates that derivatives extracted from new local models, based on few training points, are not very accurate. For the numerically estimated derivatives, figure 5.11, progress towards the desired pose is more direct, however, each full iteration requires additional robot movements for the estimation of derivatives.

\subsection{Dynamic System Control}

The final set of experiments uses LWPR to learn a controller, based on inverse dynamics, for a dynamical system, the BRIO labyrinth game. Computer vision is used to track the ball and four markers in the maze. This provides the position of the ball in the maze, independet of the motion of the maze relative to the camera. The path through the maze is known by the system and the objective is to learn a controller which enables the path to be followed. Both exploration and learning from demonstration are used to obtain training data. 


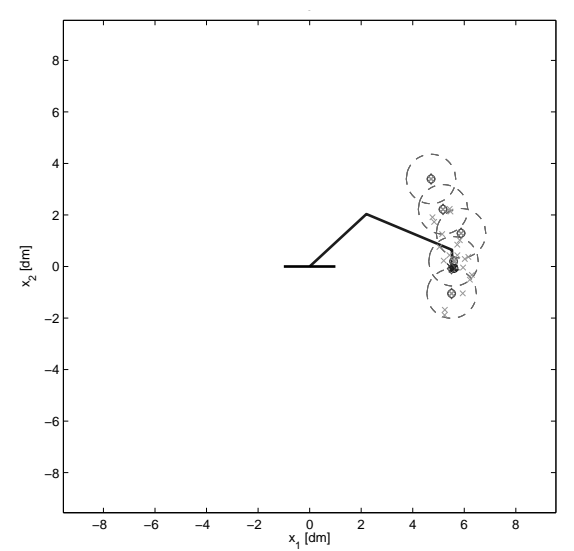

(a)

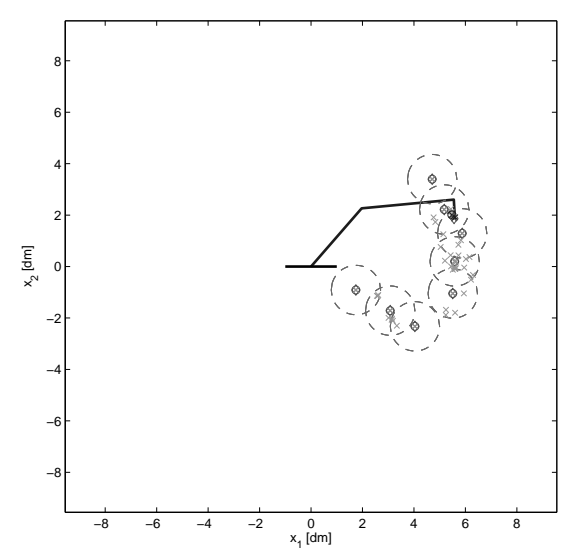

(c)

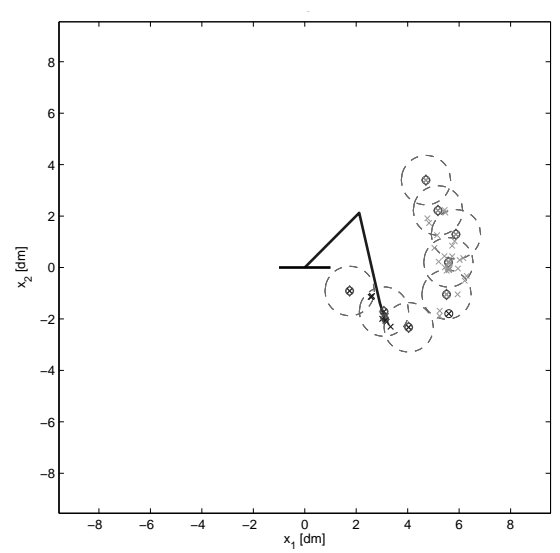

(b)

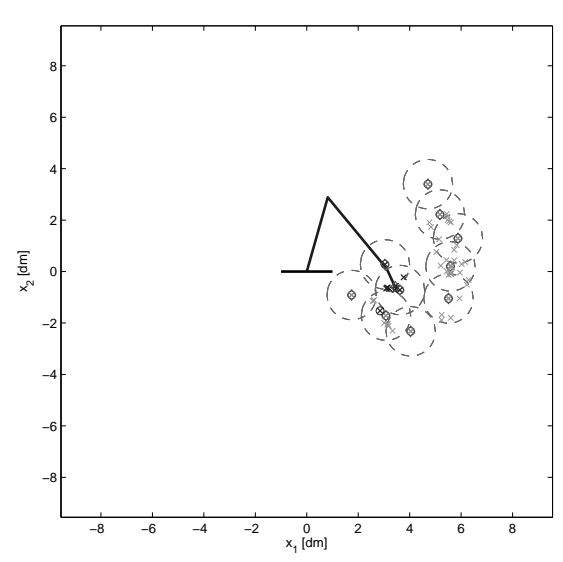

(d)

Figure 5.10: Illustration of four reaching sequences using derivatives extracted from the learned model. Visited points are marked by crosses and the valid area of each local model is marked by a circle. 


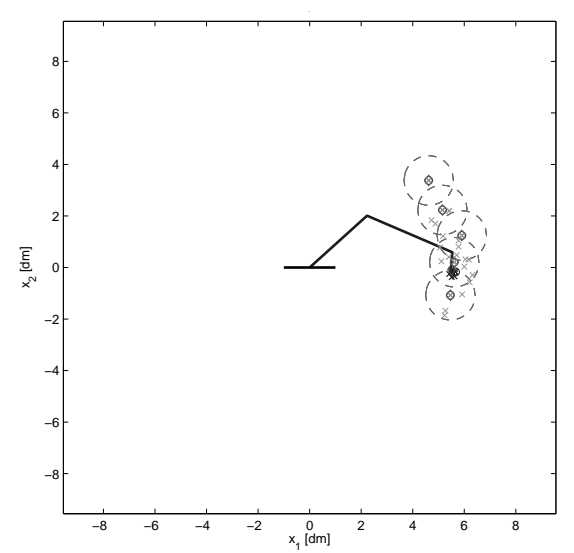

(a)

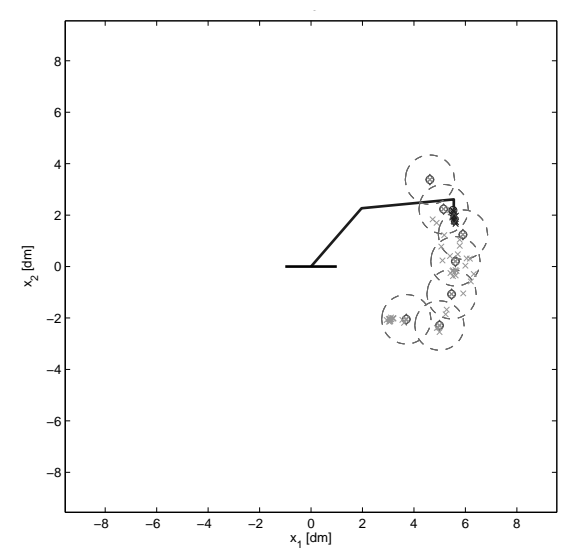

(c)

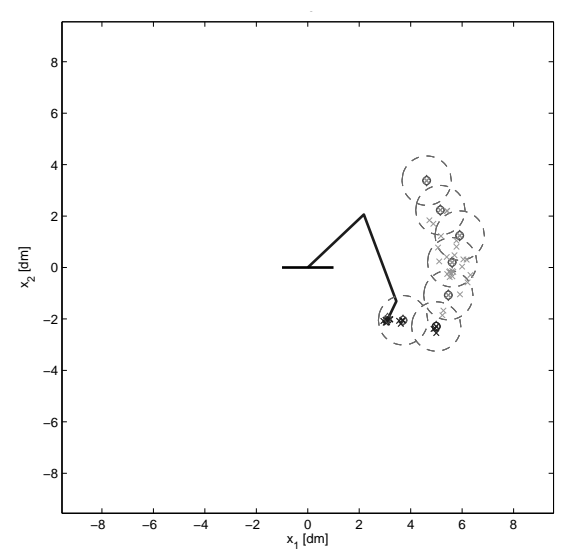

(b)

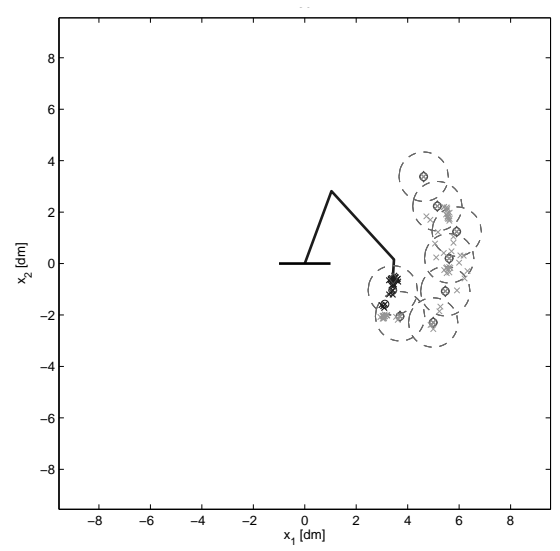

(d)

Figure 5.11: Illustration of four reaching sequences using numerically estimated derivatives. Visited points are marked by crosses and the valid area of each local model is marked by a circle. 
For a time discrete system, the inverse dynamics learning problem can be stated as learning the mapping

$$
\left(\begin{array}{l}
\mathbf{x} \\
\mathbf{x}^{+}
\end{array}\right) \rightarrow(\mathbf{u})
$$

where $\mathbf{x}$ is the current state, $\mathbf{x}^{+}$is the desired state and $\mathbf{u}$ is the predicted control signal which is supposed to bring the system from state $\mathbf{x}$ to state $\mathbf{x}^{+}$. In contrast to a regular learned system model $(\mathbf{x}, \mathbf{u}) \rightarrow \mathbf{x}^{+}$, which predicts a future state given the current state and the applied control signal. The mapping (5.1) can directly be used for controlling the system, given a desired state $\mathbf{x}^{+}$. Training data are obtained after every action, when the resulting state $\mathbf{x}^{+}$can be observed. The collection of previous state $\mathbf{x}$, the chosen action $\mathbf{u}$ and the resulting state $\mathbf{x}^{+}$then constitute a training sample. In this application, time is naturally discretized by the framerate of the camera.

Learning of the inverse dynamics is comparable to learning of the inverse kinematics of a serial robotic manipulator. The forward dynamics, disregarding disturbances, maps the current state and the applied control signal to one specific new state. Given the current state and a desired state, there may be multiple ways (different control signals) which brings the system to the desired state.

The use of learning from demonstration for this dynamical system differ in one major way from the learning from demonstration used for the autonomous driving system. In this case, the system learns the effect of an action given a certain state, independent of this effect being good or bad with respect to the goal of the game. For the autonomous driving system, the system has to predict the correct action (or at least a reasonable action) given a certain state.

This has a significant effect on the reaction to a bad teacher. In the case of autonomous driving the system will learn, and later practice, bad behavior. For the labyrinth game controller, the bad actions will be learned, but never used unless it leads to a desired state (in which case it is not a bad action). This is also apparent from the inputs to the learning systems for control signal prediction. The autonomous vehicle only requires the current state (visual feature vector) while the labyrinth controller requires both the current state and the desired state, where the latter is obtanied deterministically from the deviation from the desired path, and is not learned.

It is this difference that enables the use of exploration for training the labyrinth game controller. Simply put, if an action leading to the desired state is not known, any random action can be tried and its effect learned. If the effect is useful, the newly learned action will be used later. In this case, a hand tuned PID-controller is used for generating explorative actions.

Two LWPR based controllers have been evaluated. The first, LWPR-2, uses ball velocity as state vector, that is, assuming the dynamics of the game to be spatially invariant. The second, LWPR-4, also include the absolute ball position in the current state. 


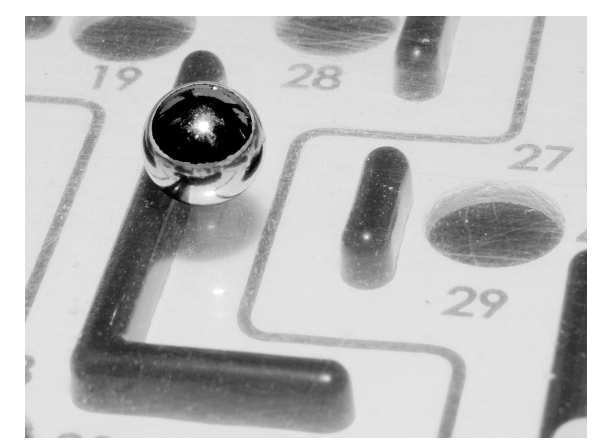

Figure 5.12: Simplified maze for control performance comparison.

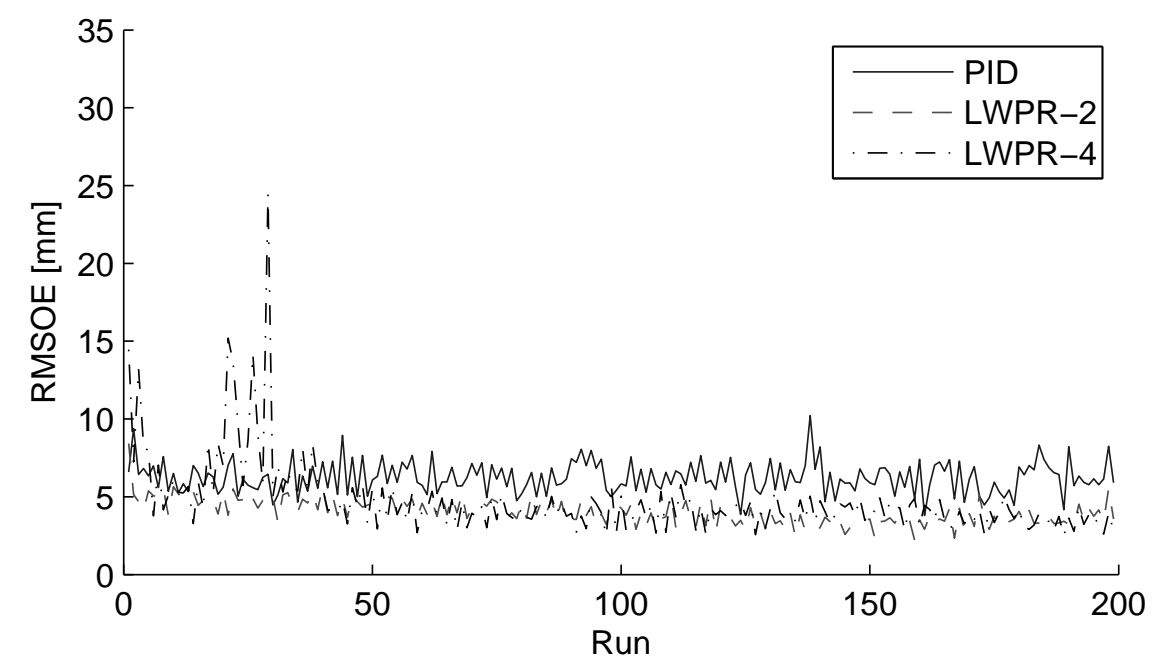

Figure 5.13: Mean path deviation versus number of runs along the desired path.

\subsubsection{Experiments in Spatially Invariant Maze}

A simplified maze was created without holes and obstacles, see figure 5.12. This greatly reduces the randomness introduced by the regular maze and increases comparability of the results by the different learning systems.

The evaluated systems are supposed to follow a generated smooth target path ${ }^{4}$. The error is calculated as the root mean squared distance from the ball to the closest part of the desired trajectory, over each run. Each system is started untrained and is allowed to continue until the desired trajectory has been completed 200 times.

The results are shown in figure 5.13. PID is the baseline system without

\footnotetext{
${ }^{4} \mathrm{~A}$ one period sinusoidal with amplitude $5 \mathrm{~cm}$ and wavelenght $15 \mathrm{~cm}$.
} 


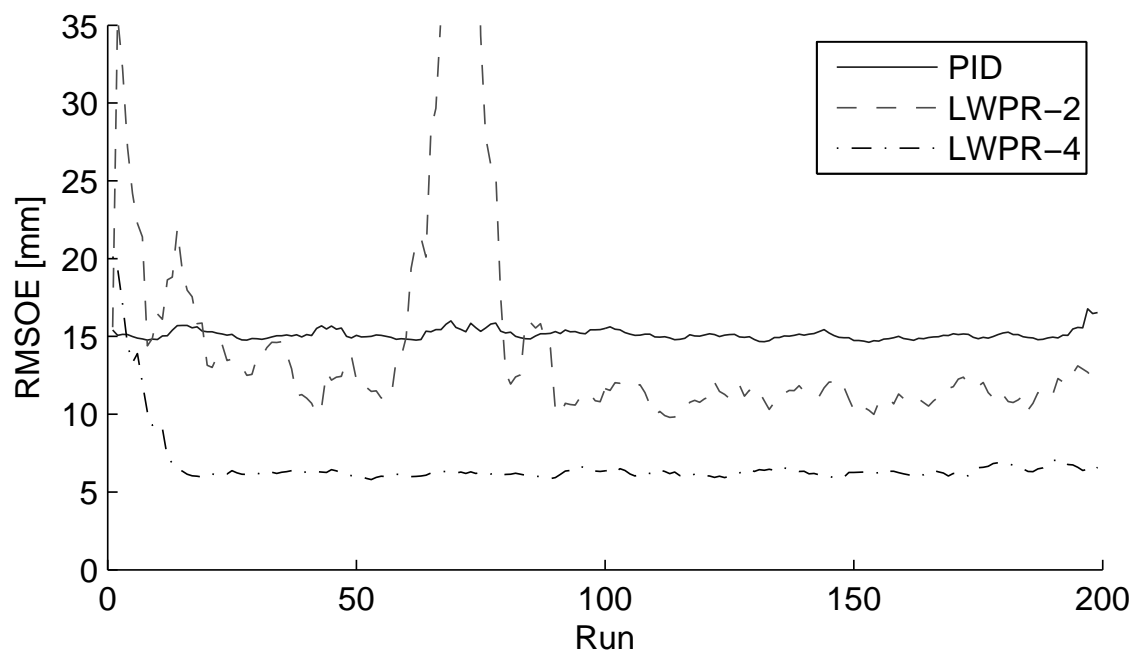

Figure 5.14: Mean path deviation versus number of runs along the desired path, spatially variant disturbance. The plot shows the mean of 10 experiments.

learning which generates the control signal in case the learning systems are unable to make a prediction. Performance varies even when the systems are sufficiently trained (later runs). What is noteworthy is that the learning systems, LWPR-2 and LWPR-4, both outperform the teacher system, PID, after some training.

The ability to handle spatially dependent disturbances is evaluated in a second experiment where a constant offset is added to the control signal when the ball is in one half of the maze. The results are shown in figure 5.14. As expected, the spatially aware learning system, LWPR-4, outperforms the others. The performance of LWPR-4 is similar to its performance in the first experiment. The decrease in error is visible when comparing early runs to later runs, see figure 5.15 where a different desired path is used.

\subsubsection{Experiment in Real Maze}

The real maze poses a greater challenge to the learning controllers. The obstacles introduces local non-linearities in ball behavior. Only the spatially aware controller was able to learn a mapping sufficient for successfull maze navigation. This at the cost of more training and higher memory demands. A reason for the failure of the other controllers is visible in the lower right corner of the top plot in figure 5.15 where the PID controller is mostly active due to lack of training data. A control mapping suitable for the open areas is not suitable for the different dynamics of the ball close to the edge of the maze. Oscillations are induced by the controller.

In the case of the real maze, learning from demonstration is particulary useful, if the teacher possesses some basic skills of playing the game. Demonstration by a skilled teacher reduces the amount of training data corresponding to actions 

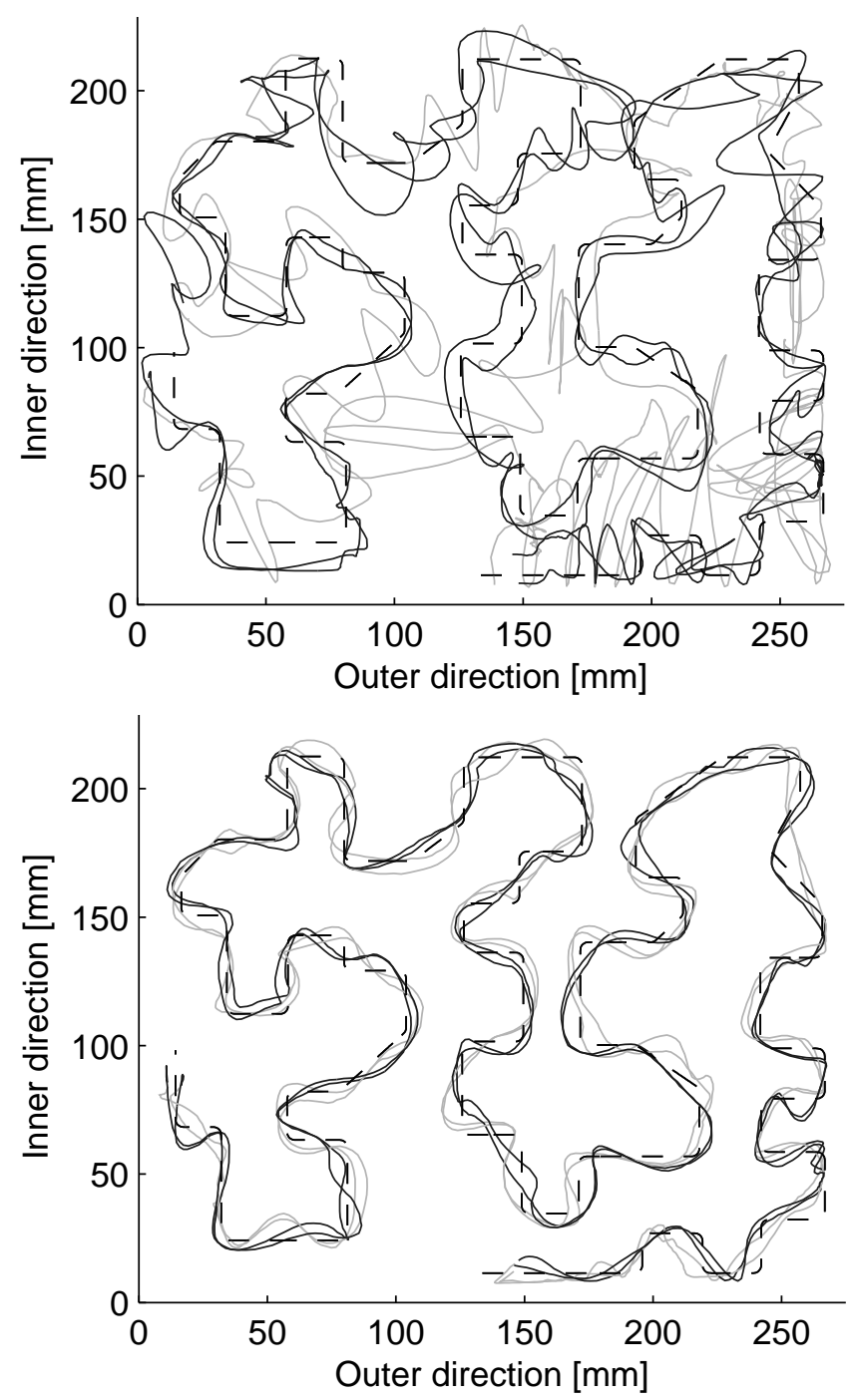

Figure 5.15: Trajectories of LWPR-4 along a more challenging path. Top: early, untrained system. Bottom: after training. The desired path is dashed. 


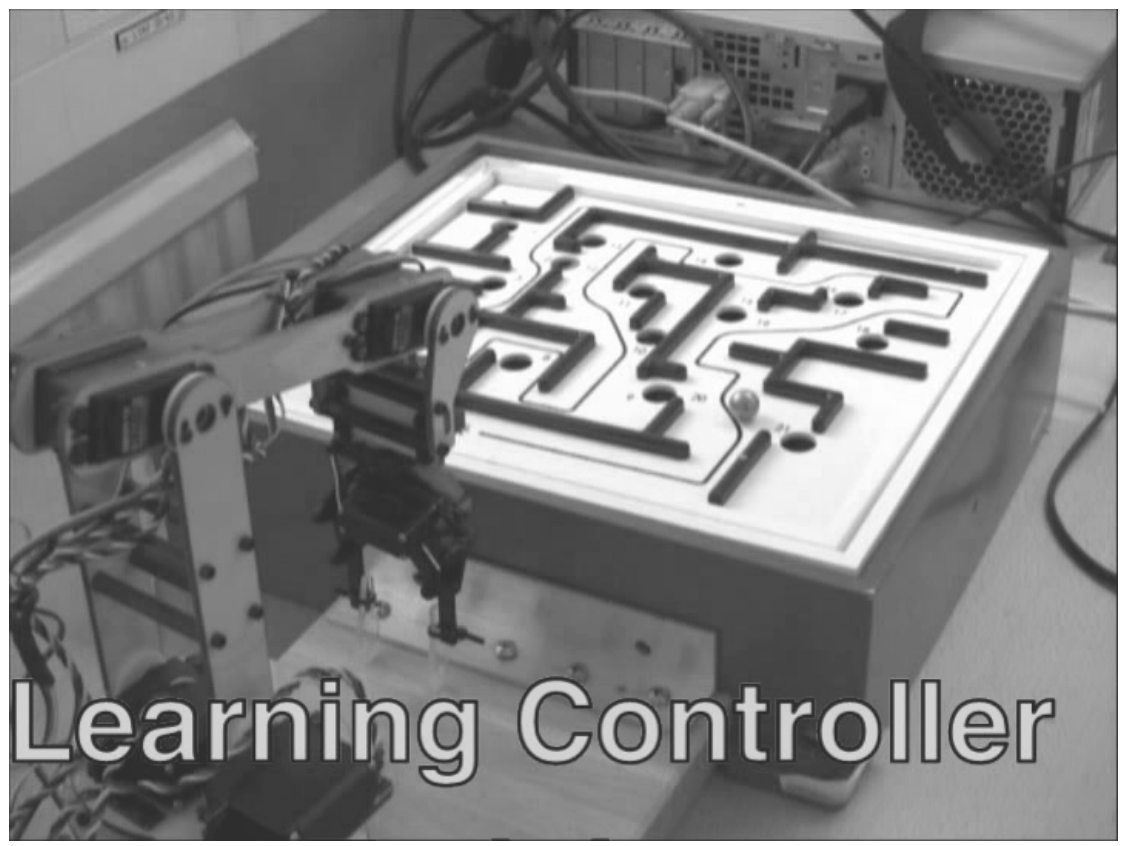

Figure 5.16: Frame from http://users.isy.liu.se/cvl/ofjall/leapwall_ii.mp4.

which will never be required, as is produced by an explorative approach. For LWPR, these learned but unused actions still generate new local models and slow down prediction. It has been noticed that after a few hours, predictions slow down enough for the delay in action to be severe enough for a noticable decay in game performance.

The supplementary video ${ }^{5}$. demonstrates the performance of the system. One frame from the video is shown in figure 5.16. The oscillatory behavior from the PID explorative controller is apparent during the first trials. After training, the ball trajectory is smoother and the control actions are smaller. At the end of the maze where less training data is available, control actions increase in amplitude and oscillations occur.

\footnotetext{
${ }^{5}$ Available at http://users.isy.liu.se/cvl/ofjall/leapwall_ii.mp4
} 


\section{Chapter 6}

\section{Conclusion and Future Research}

A selection of robotics problems have been presented along with operational demonstrations of learning systems solving these problems. Particularly, by representing input and output information using the channel representation, even simple learning algorithms can perform well. The qHebb associative learning on channel representations has illustrated this. While the learning and prediction operations are simple in the channel domain, the performance of qHebb matches the performance of the best batch learning methods in the original domain. The proposed method is online and multi-modal capable. Further, large input spaces, such as direct input from low-level visual features, are natively handled by qHebb.

The ability to handle large input spaces sets qHebb apart from ROGER. The other main competitor, LWPR, does not support multi-modal outputs and the dimensionality of the visual input is at the limit of what is possible for LWPR. While earlier associative learning metods are both online and multi-modal, it was not possible for a system to learn vision based autonomous driving without the proposed extensions that define qHebb.

Still there remain many possibilities for future research. Among the first next tasks is to analyze what is learned by means of visual heat maps, similar to those used for random forest regression [13]. There is also the possibility of inhibitory associations, that is, allowing negative coefficients in the linkage matrix.

Current approaches to machine learning strongly resemble the switchboard idea of Donald Hebb, mapping input values to output values. In the unimodal case this is function approximation. However, Hebb notes:

In mammals even as low as the rat it has turned out to be impossible to describe behavior as an interaction directly between sensory and motor processes. Something like thinking, that is, intervenes. "Thought" undoubtedly has the connotation of a human degree of complexity in cerebral function and may mean too much to be applied to lower animals. But even in the rat there is evidence that behavior is not 
completely controlled by immediate sensory events: there are central processes operating also.

- Donald Hebb, 1949 [21]

The channel representation framework allows for interesting implementations of thinking, or, allowing intervention by higher level systems. With a probabilistic view, a higher level system can affect the mapping by means of providing a channel encoded prior. In the case of the autonomous vehicle, such a prior can be used to select one mode at an intersection, while the prior can be made weak enough to avoid turning where there is no intersection. This prior expresses a desire to go in a certain direction. However, the learnt mapping will only allow this where there is an opportunity to do so.

There are further approaches used by biological systems whose technological counterparts may be useful for technical systems. One example is sleeping, using periods of system downtime for re-processing data, re-arranging, compressing or in general, assimilating, recently learnt mappings. Another example is dreaming, corresponding to offline training on hallucinated training examples generated by interpolation or extrapolation of previously seen real examples.

Still, 48 years after The Summer Vision Project [35], where Seymour Papert gave a group of summer workers the task of solving the computer vision problem, there are no signs on the horizion indicating that research within computer vision and machine learning will be finished any time soon. The examples provided by biological systems solving the vision problem keep inspiring computer vision research and as is pointed out already by Hebb, there is still no reason to believe anything else than once there is enough computing power, vision will be solved:

Modern psychology takes completely for granted that behavior and neural function are perfectly correlated, that one is completely caused by the other. There is no separate soul or life-force to stick a finger into the brain now and then and make neural cells do what they would not otherwise. Actually, of course, this is a working assumption only-as long as there are unexplained aspects of behavior.

- Donald Hebb, 1949 [21]

But while the computational capability of biological systems still is far greater than the capabilities of technical systems and while an artificial system capable of learning all aspects of driving without a strong dependence on a multitude of sensors and external information seems distant, one application seems closer at hand: learning driver assistant systems. When driving onto a long road, the system will learn the particularities of the current road and traffic situation. After some training, the system can relieve the driver of basic driving tasks. 


\section{Bibliography}

[1] Pieter Abbeel, Adam Coates, and Andrew Y Ng. Autonomous helicopter aerobatics through apprenticeship learning. The International Journal of Robotics Research, 2010.

[2] A. Baranes and P.-Y. Oudeyer. Intrinsically motivated goal exploration for active motor learning in robots: A case study. In Intelligent Robots and Systems (IROS), 2010 IEEE/RSJ International Conference on, pages 1766 -1773 , oct. 2010.

[3] Parag Batavia, Dean Pomerleau, and Chuck Thorpe. Applying advanced learning algorithms to ALVINN. Technical Report CMU-RI-TR-96-31, Robotics Institute, Pittsburgh, PA, October 1996.

[4] Leo Breiman. Bagging predictors. Machine learning, 24(2):123-140, 1996.

[5] Leo Breiman. Random forests. Machine Learning, 45(1):5-32, 2001.

[6] Chih-Chung Chang and Chih-Jen Lin. Libsvm: A library for support vector machines. ACM Trans. Intell. Syst. Technol., 2(3):27:1-27:27, 2011.

[7] William S Cleveland and Susan J Devlin. Locally weighted regression: an approach to regression analysis by local fitting. Journal of the American Statistical Association, 83(403):596-610, 1988.

[8] David A. Cohn, Zoubin Ghahramani, and Michael I. Jordan. Active learning with statistical models. CoRR, cs.AI/9603104, 1996.

[9] T.S. Collett and M.F. Land. Visual control of flight behaviour in the hoverflysyritta pipiens l. Journal of comparative physiology, 99(1):1-66, 1975.

[10] Pierre Dangauthier, Pierre Bessiere, and Anne Spalanzani. Feature Selection For Self-Supervised Learning. Rapport technique, 2005. AAAI Spring Symposium Series.

[11] Charles Darwin. On the Origin of Species by Means of Natural Selection, Or, The Preservation of Favoured Races in the Struggle for Life. J. Murray, 1859.

[12] Sophie Deneve, Peter E Latham, and Alexandre Pouget. Reading population codes: a neural implementation of ideal observers. Nature neuroscience, 2(8):740-745, 1999. 
[13] L. Ellis, N. Pugeault, K. Öfjäll, J. Hedborg, R. Bowden, and M. Felsberg. Autonomous navigation and sign detector learning. In Robot Vision (WORV), 2013 IEEE Workshop on, pages 144-151, Jan 2013.

[14] M. Felsberg, F. Larsson, J. Wiklund, N. Wadströmer, and J. Ahlberg. Online learning of correspondences between images. IEEE Transactions on Pattern Analysis and Machine Intelligence, 2013.

[15] Michael Felsberg. Incremental computation of feature hierarchies. In Michael Goesele, Stefan Roth, Arjan Kuijper, Bernt Schiele, and Konrad Schindler, editors, Pattern Recognition, volume 6376 of Lecture Notes in Computer Science, pages 523-532. Springer Berlin Heidelberg, 2010.

[16] P.-E. Forssén. Low and Medium Level Vision using Channel Representations. PhD thesis, Linköping University, Sweden, 2004.

[17] G. H. Granlund. An Associative Perception-Action Structure Using a Localized Space Variant Information Representation. In Proceedings of Algebraic Frames for the Perception-Action Cycle (AFPAC), Germany, September 2000.

[18] G. H. Granlund and A. Moe. Unrestricted recognition of 3-d objects for robotics using multi-level triplet invariants. Artificial Intelligence Magazine, $25(2): 51-67,2004$.

[19] Daniel H Grollman. Teaching Old Dogs New Tricks: Incremental Multimap Regression for Interactive Robot Learning from Demonstration. PhD thesis, Brown University, 2010.

[20] Simon S Haykin. Neural networks: a comprehensive foundation. Prentice Hall, Upper Saddle River, N.J., 1999.

[21] Donald Hebb. The Organization of Behavior. Wiley, New York, 1949.

[22] David H Hubel and Torsten N Wiesel. Receptive fields of single neurones in the cat's striate cortex. The Journal of physiology, 148(3):574, 1959.

[23] Erik Jonsson. Channel-Coded Feature Maps for Computer Vision and Machine Learning. PhD thesis, Linköping University, Computer Vision, The Institute of Technology, 2008.

[24] Stephen W Kuffler. Discharge patterns and functional organization of mammalian retina. J Neurophysiol, 16(1):37-68, 1953.

[25] Andy Liaw and Matthew Wiener. Classification and regression by randomforest. $R$ News, 2(3):18-22, 2002.

[26] Jorge J. Moré. The Levenberg-Marquardt algorithm: Implementation and theory. In G. A. Watson, editor, Numerical Analysis, pages 105-116. Springer, Berlin, 1977. 
[27] David E. Moriarty and Risto Miikkulainen. Discovering complex othello strategies through evolutionary neural networks. Connection Science, 7:195209, 1995.

[28] Kristoffer Öfjäll and Michael Felsberg. Combining vision, machine learning and automatic control to play the labyrinth game. In Proceedings of $S S B A$, Swedish Symposium on Image Analysis, Feb 2012.

[29] Kristoffer Öfjäll and Michael Felsberg. Integrating learning and optimization for active vision inverse kinematics. In Proceedings of SSBA, Swedish Symposium on Image Analysis, March 2013.

[30] Kristoffer Öfjäll and Michael Felsberg. Rapid explorative direct inverse kinematics learning of relevant locations for active vision. In Robot Vision (WORV), 2013 IEEE Workshop on, pages 14-19, Jan 2013.

[31] Kristoffer Öfjäll and Michael Felsberg. Biologically inspired online learning of visual autonomous driving. In Proceedings of the British Machine Vision Conference. BMVA Press, 2014.

[32] Kristoffer Öfjäll and Michael Felsberg. Online learning and mode switching for autonomous driving from demonstration. In Proceedings of SSBA, Swedish Symposium on Image Analysis, March 2014.

[33] Kristoffer Öfjäll and Michael Felsberg. Online learning of vision-based robot control during autonomous operation. In Yu Sun, Aman Behal, and ChiKit Ronald Chung, editors, New Development in Robot Vision. Springer, Berlin, 2014.

[34] Erkki Oja. Simplified neuron model as a principal component analyzer. Journal of Mathematical Biology, 15(3):267-273, 1982.

[35] Seymour Papert. The summer vision project. MIT AI Memos (1959-2004), 1966.

[36] Ivan Pavlov. The experimental psychology and psychopathology of animals. The 14th International Medical Congress, Madrid, Spain, 1903.

[37] A. Pouget, P. Dayan, and R. S. Zemel. Inference and computation with population codes. Annu. Rev. Neurosci., 26:381-410, 2003.

[38] N. Pugeault and R. Bowden. Driving me around the bend: Learning to drive from visual gist. In Computer Vision Workshops (ICCV Workshops), 2011 IEEE International Conference on, pages 1022-1029, Nov 2011.

[39] D. Saad. On-Line Learning in Neural Networks. Publications of the Newton Institute. Cambridge University Press, 2009.

[40] Ryo Saegusa, Giorgio Metta, Giulio Sandini, and Sophie Sakka. Active motor babbling for sensorimotor learning. In Robotics and Biomimetics, 2008. ROBIO 2008. IEEE International Conference on, pages 794 -799, feb. 2009. 
[41] S Rasoul Safavian and David Landgrebe. A survey of decision tree classifier methodology. IEEE transactions on systems, man, and cybernetics, 21(3):660-674, 1991.

[42] S. Schaal and C. Atkeson. Learning control in robotics. Robotics Automation Magazine, IEEE, 17(2):20 -29, june 2010.

[43] S. Schaal and C.G. Atkeson. Robot juggling: implementation of memorybased learning. Control Systems, IEEE, 14(1):57 -71, feb. 1994.

[44] Maria Schmiterlöw. Autonomous path following using convolutional networks. Master's thesis, Linköping University, Computer Vision, The Institute of Technology, 2012.

[45] David W. Scott. Averaged shifted histograms: Effective nonparametric density estimators in several dimensions. Annals of Statistics, 13(3):1024-1040, 1985 .

[46] Burr Settles. Active learning literature survey. Technical report, 2009.

[47] H. P. Snippe and J. J. Koenderink. Discrimination thresholds for channelcoded systems. Biological Cybernetics, 66:543-551, 1992.

[48] Andrew D. Straw, Serin Lee, and Michael H. Dickinson. Visual control of altitude in flying drosophila. Current Biology, 20(17):1550 - 1556, 2010.

[49] Harry L. Van Trees, Kristine L. Bell, and Zhi Tian. Detection estimation and modulation theory. Second Edition. John Wiley \& Sons, Hoboken, New Jersey, 2013.

[50] Sethu Vijayakumar, Aaron D'souza, and Stefan Schaal. Incremental online learning in high dimensions. Neural Comput., 17:2602-2634, December 2005.

[51] R. S. Zemel, P. Dayan, and A. Pouget. Probabilistic interpretation of population codes. Neural Computation, 10(2):403-430, 1998. 


\section{Part II}

\section{Publications}





\section{Publications}

The articles associated with this thesis have been removed for copyright reasons. For more details about these see:

http://urn.kb.se/resolve?urn=urn:nbn:se:liu:diva- 110892 